\title{
Identification of the Prognostic LncRNA Biomarkers and Comprehensive Analysis of LncRNA-Mediated ceRNA Network for Uterine Corpus Endometrial Carcinoma
}

\author{
Yiran Cai \\ Medical School of Nantong University \\ Jin Cui \\ Medical School of Nantong University \\ Huiqun Wu ( $\nabla$ wuhuiqun@ntu.edu.cn ) \\ Medical School of Nantong University
}

\section{Research Article}

Keywords: Uterine corpus endometrial cancer, prognostic biomarker, long non-coding RNA, competing endogenous RNA network, The Cancer Genome Atlas(TCGA), Clinical Proteomic Tumor Analysis Consortium(CPTAC)

Posted Date: November 8th, 2021

DOl: https://doi.org/10.21203/rs.3.rs-989585/v1

License: (c) (i) This work is licensed under a Creative Commons Attribution 4.0 International License. Read Full License 


\section{Abstract \\ Background}

Given that long non-coding RNAs (IncRNAs) involved in the tumor initiation or progression of the endometrium and that competing endogenous RNA (ceRNA) plays an important role in increasingly more biological processes, IncRNA-mediated ceRNA is likely to function in the pathogenesis of uterine corpus endometrial carcinoma (UCEC). Our present study aimed to explore the potential molecular mechanisms for the prognosis of UCEC through an IncRNA-mediated ceRNA network.

\section{Methods}

The transcriptome profiles and corresponding clinical profiles of UCEC dataset were retrieved from CPTAC and TCGA databases respectively. Differentially expressed genes (DEGs) in UCEC samples were identified via "Edge R" package. Then, an integrated bioinformatics analysis including functional enrichment analysis, tumor infiltrating immune cell(TIIC) analysis, Kaplan-Meier curve, Cox regression analysis were conducted to analyze the prognostic biomarkers.

\section{Results}

In the CPTAC dataset of UCEC, a ceRNA network comprised of 36 miRNAs, 123 IncRNAs and 124 targeted mRNAs was established, and 8 of 123 prognostic-related DElncRNAs(Differentially Expressed long noncoding RNA) were identified. While in the TCGA dataset, a ceRNA network comprised of 38 miRNAs, 83 IncRNAs and 110 targeted mRNAs was established, and 2 of 83 prognostic-related DElncRNAs were identified. After filtered by risk grouping and Cox regression analysis, 10 prognostic-related IncRNAs including LINC00443, LINC00483, C2orf48, TRBV11-2, MEG-8 were identified. In addition, 33 survivalrelated DEmRNAs(Differentially Expressed messager RNA) in two ceRNA networks were further validated in the HPA database. Finally, six IncRNA/miRNA/mRNA axes were established to elucidate prognostic regulatory roles in UCEC.

\section{Conclusion}

Several prognostic IncRNAs are identified and prognostic model of IncRNA-mediated ceRNA network is constructed, which promotes the understanding of UCEC development mechanisms and potential therapeutic targets.

\section{Introduction}

Uterine corpus endometrial cancer (UCEC), one of globally common gynecological malignancies, presents a possibly upward trend of with the increase of obese women. [1-3]The choice for treatment of UCEC has 
considerable exploration and development prospects for the perspective of molecular biology. In previous studies, risk factors including p53 expression[4], and estrogen receptor (ER) and progesterone receptor (PR) expression[5], as well as clinical treatment manners have been identified[6]. The discovery of these factors provides access to take advantage of underlying therapeutic biomarkers for personalized treatment strategies.MiRNA is a family of small non-coding RNA molecules of about 21 to 25 nucleotides long. miRNA inhibits translation of targeted mRNA or affects its stability by specific identifications, and down regulates its expression by combining at its 3'UTR site[7]. The abnormal miRNA expression in development of tumors has been confirmed by many studies [8]. LncRNA is known to play a role as key signal transduction mediators in the occurrence, progression and treatment of numerous malignancies[9-11]. According to the ceRNA hypothesis[12], IncRNA is a molecular sponge of miRNA, which suppresses the activity of miRNA by binding with microRNA response element (MRE) and down regulates the expression of the target genes indirectly. Based on this argument, the ceRNA network has been extensively researched and verified in lung cancer[13], breast cancer[14] and so on, while there have been little discussion on IncRNA-mediated ceRNA networks of UCEC.

Therefore, in this study, we retrieved and analyzed IncRNA expression in UCEC from TCGA and CPTAC database separately and performed an integrated bioinformatics analysis including functional enrichment analysis, Tumor Infiltrating Immune Cell (TIIC) analysis, and constructed the UCEC-specific ceRNA network and figured out the underlying association between those ceRNAs and the progression of UCEC.

\section{Methods}

\section{Data Collection}

Transcriptional and clinical data of UCEC in both The Cancer Genome Atlas data portal(TCGA; https://portal.gdc.cancer.gov/) and Clinical Proteomic Tumor Analysis Consortium(CPTAC; https://cptacdata-portal.georgetown.edu/data-use-agreement) were retrieved. There were 587 samples downloaded from TCGA, containing 555 UCEC patients and 32 normal specimens, and 116 samples downloaded from CPTAC, containing 101 UCEC patients and 15 normal specimens, contributing to the UCEC and normal control group as a cohort. The clinical features of UCEC patients from 2 databases were respectively shown (Table 1). 12 other samples of originally 555 UCEC samples in TCGA database were omitted in this whole study due to missing associated information. Therefore, actually a total of 575 samples from TCGA were conducted to the further analyses. The clinical features of UCEC patients including age, gender, race, pathology stage, histological type and vital status were extracted. Transcriptome data were annotated with the Genecode website (https://www.gencodegenes.org/). No samples were excluded when to screen for differentially expressed RNAs (DERNAs, including three ones: differentially expressed long noncoding RNA(DElncRNA), differentially expressed microRNA (DEmiRNA), differentially expressed messager RNA(DEmRNA)). Both databases were publically available and were released in compliance with ethical approvals; therefore no further application from University Ethics Committee was obtained. 
Table 1

Characteristics of 543 UCEC patients from TCGA and 101 UCEC patients from CPTAC database

\begin{tabular}{|c|c|c|}
\hline TCGA features & Variables & Patients (n, \%) \\
\hline \multirow[t]{3}{*}{ Age } & $>60$ & $334(61.51)$ \\
\hline & $\leq 60$ & 206(37.94) \\
\hline & NA & $3(0.55)$ \\
\hline Gender & Female & $543(100.00)$ \\
\hline \multirow[t]{6}{*}{ Race } & White & $372(68.51)$ \\
\hline & NA & $32(5.89)$ \\
\hline & Black or African American & 106(19.52) \\
\hline & Native Hawaiian or other pacific islander & $9(1.66)$ \\
\hline & American indian or Alaska native & $3(0.55)$ \\
\hline & Asian & $20(3.68)$ \\
\hline \multirow[t]{4}{*}{ Pathology stage } & Stage I & $339(62.43)$ \\
\hline & Stage II & $51(9.39)$ \\
\hline & Stage III & 124(22.84) \\
\hline & Stage IV & $29(5.34)$ \\
\hline \multirow[t]{3}{*}{ Primary diagnosis } & Endometrioid carcinoma & $399(73.48)$ \\
\hline & Serous cystadenocarcinoma & 133(24.49) \\
\hline & Other types & $11(2.03)$ \\
\hline \multirow[t]{2}{*}{ Survival } & Alive & $452(83.24)$ \\
\hline & Dead & $91(16.76)$ \\
\hline CPTAC features & Variables & Patients (n, \%) \\
\hline \multirow[t]{2}{*}{ Age } & $>60$ & $64(63.37)$ \\
\hline & $\leq 60$ & $37(36.63)$ \\
\hline Gender & Female & 101(100.00) \\
\hline \multirow[t]{3}{*}{ Race } & White & $59(58.42)$ \\
\hline & NA & $38(37.62)$ \\
\hline & Black or African American & $3(2.97)$ \\
\hline
\end{tabular}




\begin{tabular}{|c|c|c|}
\hline \multirow[t]{2}{*}{ TCGA features } & Variables & Patients (n, \%) \\
\hline & Asian & $1(0.99)$ \\
\hline \multirow[t]{4}{*}{ Pathology stage } & Stage I & $75(74.26)$ \\
\hline & Stage II & $8(7.92)$ \\
\hline & Stage III & $15(14.85)$ \\
\hline & Stage IV & $3(2.97)$ \\
\hline \multirow[t]{2}{*}{ Histological type } & Endometrioid carcinoma & $85(84.16)$ \\
\hline & Other types & $16(15.84)$ \\
\hline \multirow[t]{3}{*}{ Tumor grade } & $\mathrm{G} 1+\mathrm{G} 2$ & $71(70.30)$ \\
\hline & G3 & $27(26.73)$ \\
\hline & Other & $3(2.97)$ \\
\hline \multirow[t]{3}{*}{ Survival } & Alive & $84(83.17)$ \\
\hline & Dead & $10(9.90)$ \\
\hline & Not reported & $7(6.93)$ \\
\hline
\end{tabular}

\section{Identification of DEGs}

The "edge R" was utilized to identify differentially expressed genes (DEGs) between the normal samples and UCEC patients by the criteria (false discovery rate (FDR) or adjusted $\mathrm{P}<0.01$, and $\left|\log _{2} F C\right|>2$ (FC is fold change)). All of the statistical tests were conducted and the heatmap and volcano plot were displayed by ggplot2 package in R software package (version:4.0.3; https://www.r-project.org/) and statistical significance was defined as a P-value $<0.05$ unless otherwise stated.

\section{Construction of Protein-Protein Interaction Network}

In this study, a total of 1064 and 917 DEGs filtered by $\left|\log _{2} F C\right|>3$ from the two databases were subjected to perform protein-protein interaction(PPI) network analysis using Search Tool for the Retrieval of Interacting Genes (STRING; https://string-db.org/)[15]. An interaction with a combined score by default $>0.4$ was considered statistically significant. Cytoscape (version 3.8.2) is a bioinformatics software platform publically used for visualizing molecular interaction networks [16]. To find hub genes actively participated in UCEC progression, we employed the maximal clique centrality (MCC) algorithm to represent 20 key mRNAs with important biological functions via CytoHubba in Cytoscape[17].

\section{Tumor Infiltrating Immune Cells Profiling}


To characterize proportions of tumor infiltrating immune cells(TIICs) in UCEC, CIBERSORT (http://cibersort. stanford. edu/) algorithm in combination with a LM22 gene signature matrix was used to assess the relative fractions of 22 invasive immune cell subtypes in each UCEC sample. Regarding the results of the algorithm, we also accumulated the percentage of each immune cell theoretically calculated from each patient sample and presented those top ranked immune cell types in bar graphs. Worthy to point out, if these UCEC samples lack of associated transcriptome information, they were then omitted. Additionally, to investigate the immune infiltration landscape of UCEC, gene set enrichment analysis (ssGSEA) was performed by GSVA package in R (https://bioconductor.org/ packages/release/bioc/html/GSVA.html) to calculate the score of immune infiltration in each sample on the basis of immune cell-specific gene expression levels. Standardized profiles of gene expression data in both groups were extracted and immune scores were evaluated, scoring types including different cell clusters and respective expression values corresponding to different colors.

\section{Construction of ceRNA Network and Extraction of Survival-related LncRNA-miRNA-mRNA subnetwork}

In this study, the IncRNA-mediated ceRNA(competing endogenous RNA) network of UCEC was constructed as IncRNA-miRNA and miRNA-mRNA pairs. To build a ceRNA network, following steps were conducted. Firstly, based on miRNAs provided by the miRcode website (http://www.mircode.org), IncRNAtargeted miRNAs simultaneously in our DEmiRNAs were filtered. Secondly, to identify the miRNA-targeted mRNAs, we searched miRTarBase[18] (https://mirtarbase.cuhk.edu.cn/ miRTarBase/miRTarBase_2019/php/index.php), miRDB[19] (http://www.mirdb.org/), TargetScan[20] (http://www.targetscan.org/) in combination to obtain targeted mRNAs. Finally, Cytoscape software v3.8.2 (https://cytoscape.org/) was adopted to visualize the UCECrelated ceRNA network. The paired interactions in above network were analyzed in two UCEC datasets from TCGA and CPTAC, respectively. Subsequently, we took the intersection of the two ceRNA networks.

Furthermore, on the foundation of overall ceRNA network, we firstly conducted survival analyses and Cox regression analyses of these genes, both results of which were considered as hub genes in our ceRNA network. Thereafter, we input these hub genes to visualize their IncRNA-miRNA-mRNA regulatory relationships via Cytohubba (http://hub.iis.sinica.edu.tw/cytohubba) in Cytoscape. Eventually, we constructed a novel ceRNA subnetwork composed of IncRNA-miRNA-mRNA pairs, which may provide prognostic molecular values.

\section{Survival Analysis and LncRNA-mediated Prognostic Model Construction}

On one hand, survival $\mathrm{R}$ was operated for survival analysis of all DERNAs in the ceRNA network. KaplanMeier (K-M) curves were plotted with DERNAs by log-rank test. On the other hand, we identified the IncRNAs linked with total survival $(p<0.05)$ to act as prognostic IncRNA signature candidates and then imported them for Cox regression analyses. According to the median risk score, the UCEC patients were divided into the high risk and low risk groups. To evaluate the accuracy of models survival-related DElncRNAs, we carried out the receiver operating characteristic (ROC) curve analysis (UCEC data in CPTAC using 3 years as the predicted time, UCEC data in TCGA using 5 years as the predicted time), 
along with the area under the receiver operating characteristic curve (AUC) analysis at a criterion of AUC > 0.7. Besides, we further retrieved analyses of survival-related IncRNAs in the GEPIA database(GEPIA; http://gepia.cancer-pku.cn/index.html) by $p$ values $\leqq 0.1$ (although 0.11 was also considered as significant in this study).Subsequently, we compared these results of survival-related IncRNAs with our analyses thus to validate and prove its reliability.

\section{Validation of Dysregulated mRNAs via HPA and GEPIA databases}

The Human Protein Atlas Portal (HPA) (www.proteinatlas.org)[21] which contains different genes in specific cancer types and publicly available information of Immunohistonchemistry $(\mathrm{IHC})$ staining was used for survival analyses. Gene Expression Profiling Interactive Analysis, an interactive website, composed of 9736 patients and 8587 normal samples from TCGA and GTEx projects (The GenotypeTissue Expression project) were utilized for the analysis of RNA sequencing expression. According to prognostic IncRNA-mRNA or mRNA-miRNA signatures in the IncRNA-mediated network, we took 33 mRNAs resulted from UCEC data of TCGA into two external databases mentioned above for further retrieval and analysis.

\section{Functional and Pathway Enrichment Analysis}

To analyze functions represented in two profiles of identified DEmRNAs in the ceRNA network, Gene Ontology (GO), Kyoto Encyclopedia of genes and Genomes (KEGG) were performed by cluster Profiler $\mathrm{R}$ package and plotted by GO plot R package and KEGG plot R package. Three methods were utilized to enrich meaningful biological pathways by the standard of $p$ valve less than 0.05 .

\section{Results}

\section{Differentially expressed RNAs in UCEC}

In a study of 575 UCEC samples collected from TCGA (http://cancergenome.nih. gov/publications/publication guide lines), "edge R" (Adjust $\mathrm{P}<0.01$ and $\left|\log _{2} F C\right|>2$ ) was adjusted to identify the DERNAs, including DElncRNAs, DEmiRNAs, DEmRNAs. A total of 1513 up-regulated DEmRNAs, 914 down-regulated DEmRNAs, 686 up-regulated DElncRNAs, 330 down-regulated DElncRNAs, 124 up-regulated DEmiRNAs, and 50 down-regulated DEmiRNAs were identified in TCGA database. Similarly, a total of 116 samples were identified with the same criteria in the CPTAC database, including 2463 differential DEmRNAs, 1741 DElncRNAs and 204 DEmiRNAs. The heatmap and volcano plot of those highest dysregulated DElncRNAs, DEmiRNAs and DEmRNAs were shown in Fig. 1 and the top 10 up and down regulated DElncRNAs, DEmiRNAs and DEmRNAs were shown in supplement Tables 1.

\section{Construction of PPI Network}

The 1981 DEmRNAs ( $\log _{2} F C \mid>3$ ) were further selected to construct PPI network to select hub genes that play crucial roles in UCEC genesis. Given the large number of DEmRNAs in this module, we used 
MCC algorithm in "Cytohubba" of Cytoscape software to visualize and select hub genes in the PPI network. The top 20 high score genes in in CPTAC were shown (Fig.2A). Similarly, the top 20 high score genes in TCGA belong to Histone cluster $1 \mathrm{H}$ family which was shown (Fig.2B).

Two graphs separately describe the top 20 most dynamic hub genes and their intersection relationships evaluated by MCC algorithm in "Cytohubba". These sub-graphs of these selected mRNA-coding protein nodes are shown from highly essential (red) to essential (yellow).

\section{TIICs Enrichment Analysis}

Using CIBERSORT algorithm, we evaluated 101 tumor transcriptome profiles from CPTAC database and 543 tumor transcriptome profiles from TCGA database (Fig.3A,3B). In addition, we also performed ssGSEA analysis by GSVA package to score the corresponding TIICs in each simple sample, and finally we found that TIICs in both CPTAC and TCGA database sources expressed well (Fig.3C,3D). On account of the top five immune cell components in UCEC patients by CIBERSORT algorithm, 2 bar graphs were then visualized. In CPTAC, there were abundant CD8 T cells (28.5\%) and plasma cells $(20.5 \%)$ and other TIICs. In TCGA samples, naive CD4 cells (18.9\%) and CD8 cells (15.2\%) were well infiltrated, accompanied by slightly increased activated NK cells $(10.2 \%)$, plasma cells $(9.1 \%)$ and macrophages $\mathrm{M} 0(7.5 \%)$ (Fig.3E,3F).

\section{Construction of ceRNA Network and hub LncRNA-miRNA-mRNA subnetwork}

In order to better comprehend the interactions of mRNAs, IncRNAs, and miRNAs in UCEC, we constructed an IncRNA-mediated ceRNA regulatory network. To begin with, 1741 DElncRNAs in CPTAC database succeeded to match with 123 IncRNAs in the miRCODE database. Considering 123 of 1741 DEIncRNAs could interact with DEmiRNAs, 36 miRNAs in both miRCODE and CPTAC database were selected to construct IncRNA-miRNA pairs. Meanwhile, to interplay with 204 DEmiRNAs acquired from CPTAC database, we retrieved 1420 mRNAs in three databases (miRTarBase, miRDB and TargetScan). The 1420 miRNA-targeted mRNAs predicted in these databases were intersected with 2463 DEmRNAs thus to obtain 124 miRNA-targeted mRNAs belonging to CPTAC database. Finally, an IncRNA-mediated ceRNA network consisting of 36 miRNAs, 123 IncRNAs and 124 mRNAs were achieved (Fig.4A). Meanwhile, the same workflow of UCEC-specific ceRNA network construction was repeated in data from TCGA. We obtained an IncRNA-mediated ceRNA network consisting of 38 miRNAs, 83 IncRNAs and 110 mRNAs (Fig.4B).

Furthermore, cytohubba was applied to visualize our extracted hub genes composing of IncRNA, miRNA and mRNAs and derived regulatory ceRNA network thus to identify potentially prognostic molecular pathways of UCEC in CPTAC and TCGA (Fig.4C,4D). A total of 6 hub IncRNA-miRNA-mRNA regulatory relationships from 2 databases were shown (Table 2). Moreover, coincident ceRNA results in the overall ceRNA network from both CPTAC and TCGA databases were shown in the Venn diagram (Fig.4E). 
Table 2 CeRNA subnetwork of prognostic regulatory DEGs in UCEC from CPTAC (up) and TCGA (down) database

\begin{tabular}{|lll|}
\hline LnCRNA & miRNA & mRNA \\
\hline TRBV11-2 & has-mir-363 & SOX11 \\
\hline MEG8 & has-mir-424 & CCNE1, CBX2 \\
\cline { 2 - 3 } & has-mir-363 & SOX11 \\
\cline { 2 - 3 } & has-mir-183 & DLX4, NR3C1 \\
\hline IncRNA & miRNA & mRNA \\
\hline LINC00443, C2orf48, LINC00483 & miR-183 & DLX4, NR3C1 \\
\hline DGCR5 & has-mir-195 & CCNE1 \\
& has-mir-383 & \\
\hline & has-mir-424 & \\
\hline
\end{tabular}

\section{Identification and Validation of Prognostic IncRNAs in ceRNA networks}

In order to figure out the effects of interactions for survival between IncRNAs, miRNAs and mRNAs, we imported survival-related data of UCEC and genes in ceRNA to analyze its prognosis. Survival R were operated for DERNAs significantly correlated with overall survival in the ceRNA network $(p<0.05)$, the results of which were plotted by Kaplan-Meier (K-M) curves (Fig.5A-5W).

As shown in CPTAC, 4 survival-related DElncRNAs on the level of 3-year survival were identified in DElncRNA-mediated ceRNA networks, including FREM2-AS1, HPYR1, LINC00028, MIR205HG (Fig.5A-5D). Similarly, 19 survival-related DElncRNAs, 9 DEmiRNAs and 33 DEmRNAs on the level of 5-year survival were revealed in the ceRNA network for TCGA. Fig.5E-5W illustrated Kaplan-Meier curve analyses about 10 IncRNAs of 19 survival-related DElncRNAs, 5 mRNAs of 33 DEmRNAs and 2 of 9 DEmiRNAs derived from TCGA database. Besides, we successfully validated 5 survival-related IncRNAs in GEPIA database by $p$ values $\leqq 0.1$ (Fig.6A-6E).

To further identify DElncRNAs with prognostic features in a more accurate way, multi-Cox regression analyses and corresponded ROC curves were carried out. After eliminating some samples lacking in survival time, 94 complete samples in CPTAC were divided into the high-risk $(n=47)$ and low-risk $(n=47)$ groups (cutoff value $=-0.78$ ) and 543 samples with complete survival information in TCGA into the highrisk $(n=272)$ and low-risk $(n=272)$ cohort by median value (cutoff value $=-0.18$; one sample of survival data was just in the median and counted in both groups). We performed a multi-factor COX regression analysis and a global survival analysis of the model thus separately identified two IncRNAs of 3-year 
survival data in CPTAC and eight IncRNA prognosis candidates of 5-year survival UCEC data in TCGA by $p<0.05$ (Fig.7A,7B,7C,7F)). Receiver operating characteristic (ROC) curves tested the influence on their IncRNA signatures associated with overall survival in UCEC. Area under ROC curve of 3-year survival rate (AUC) and 5-year survival rate (AUC) were respectively 0.967 and 0.751. (Fig.7B,7E) Besides, multivariate cox regression analysis of totally 10 prognostic IncRNAs associated with overall survival in UCEC patients generated from 2 databases were shown in Table 3.

Table 3 Multivariate Cox regression analysis of totally 10 prognostic IncRNAs associated with overall survival (OS)

\begin{tabular}{|llllll|}
\hline LnCRNA(in TCGA) & Coef & HR & 95\%Cl_LL & $95 \%$ Cl_HL & p-value \\
\hline FAM41C & 0.017169325 & 1.017317565 & 1.007489748 & 1.027241249 & $5.27 \mathrm{E}-04$ \\
\hline MIR7_3HG & 0.003483874 & 1.00348995 & 1.001209451 & 1.005775643 & 0.002688886 \\
\hline LINC00483 & 0.010635045 & 1.010691798 & 1.003527131 & 1.017907617 & 0.003389722 \\
\hline ABHD11_AS1 & $8.26 \mathrm{E}-04$ & 1.000826495 & 1.00038596 & 1.001267224 & $2.35 \mathrm{E}-04$ \\
\hline LINC00443 & 0.007147984 & 1.007173592 & 1.001433816 & 1.012946267 & 0.014233205 \\
\hline OXCT1_AS1 & 0.026906491 & 1.027271739 & 1.006299256 & 1.048681314 & 0.010568912 \\
\hline PRICKLE2_AS2 & 0.273785608 & 1.314932861 & 1.133483915 & 1.525428288 & $3.02 \mathrm{E}-04$ \\
\hline GLIS3_AS1 & $9.53 \mathrm{E}-04$ & 1.000953853 & 1.000210868 & 1.001697389 & 0.011853011 \\
\hline LncRNA(in & Coef & HR & $95 \% C l \_L L$ & $95 \% C l \_H L$ & p-value \\
\hline CPTAC) & & & & & \\
\hline MEG8 & 0.007280976 & 1.007307546 & 1.003839185 & 1.010787892 & $3.51 \mathrm{E}-05$ \\
\hline TRBV11_2 & 0.027816351 & 1.028206838 & 1.012679086 & 1.043972683 & $3.40 \mathrm{E}-04$ \\
\hline
\end{tabular}

In the multivariate Cox regression analysis derived from TCGA database, 8 IncRNAs including FAM41C, MIR7_3HG, LINC00483, ABHD11_AS1, LINC00443, OXCT1_AS1, PRICKLE2_AS2 and GLIS3_AS1 were identifed to construct the OS prediction model. OS-related prediction model $=\left(0.017169325^{\star}\right.$ expression value of FAM41C)+(0.003483874* expression value of MIR7_3HG)+(0.010635045* expression value of LINC00483)+(8.26E-04* expression value of ABHD11_AS1)+(0.007147984* expression value of TRBV11_2)+(0.027816351* expression value of TRBV11_2 $)+(0.027816351$ * expression value of LINC00443)+(0.026906491* expression value of OXCT1_AS1)+(0.273785608* expression value of PRICKLE2_AS2)+(9.53E-04* expression value of GLIS3_AS1). We divided the 543 UCEC cases into the high and low-risk groups according to the median values of the OS-related prediction model. 
In the multivariate Cox regression analysis derived from CPTAC database, 2 IncRNAs including MEG8 and TRBV11_2 were identified to construct the OS prediction model. OS-related prediction model $=\left(0.007280976\right.$ * expression value of MEG8) $+\left(0.027816351^{*}\right.$ expression value of TRBV11_2). We divided the 94 UCEC cases into the high and low-risk groups according to the median values of the OS-related prediction model.

\section{Validations of survival analysis and mRNA Expression at the Transcriptional Level}

To further demonstrate the prognostic significance of 33 mRNAs screened from the ceRNA network, we selected external databases for survival analysis and validation with IHC images. Firstly, we input 33 screened mRNAs into HPA database (version 20.1; https://www.Proteinatlas.org/about/assays+annotation\#tcga_survival) to validate whether they were associated with the prognosis of UCEC. Consequences revealed that 8 mRNAs(CBX2, CCL22, CCNE1, DLX4, IGFBP5, NR3C1, SOX11, POLQ) highly expressed in UCEC were closely related with its prognosis $(\log$ rank $P$ values $₫ 0.001)$. Subsequently, we retrieved overall survival analyses of 8 mRNAs generated from GEPIA by filtered criteria of $P$ values $\leq 0.1$ (although 0.11 was also considered as significant in this study) and verified 5 mRNAs (CCNE1, CCL22, NR3C1, IGFBP5 and POLQ).

Based on two previous steps for external verifications, two IHC images of the last screened mRNAs (CCNE1, NR3C1) in the HPA database approved the same results (Fig.8A). Survival validations of 5 mRNAs including CCNE1, CCL22, NR3C1, IGFBP5 and POLQ from GEPIA were shown in the Fig.8B-8F. In this study, we identified 5 survival-related mRNAs, there were no related IHC samples of CCL22, IGFBP5 and POLQ but CCNE1 and NR3C1 to further validate in the HPA database. The translational expression level of CCNE1 and NR3C1 was positively linked with disease status, as they were up-regulated in UCEC samples.

\section{Enrichment Analyses of Functional Pathways}

To elucidate the biological functions represented in two profiles of identified DEmRNAs, we performed enrichment analyses mainly by "cluster profiler", with the standard of $p<0.05$. In this study, GO analyses disclosed that top significant GO terms $(p<0.05)$ commonly obtained from UCEC data in CPTAC (Fig.9A,9B) and TCGA database (Fig.9E,9F). The KEGG analyses revealed that what closely related to DEmRNAs originated from CPTAC were mainly enriched in pathways such as "cellular senescence", "proteoglycans in cancer" and "microRNAs in cancer" (Fig.9C,9D)). The KEGG results derived from DEmRNAs in TCGA database were shown (Fig.9G,9H). The top $20 \mathrm{GO}$ and KEGG results for TCGA and CPTAC database were provided in supplement Table 2.

\section{Discussion}

Recently, with the increase of obese women, UCEC has become one of the leading gynecologic tumors[22]. Although some diagnostic markers like CA125, CA199, and CEA are clinically used, survival results are not optimistic after routine diagnosis and therapy. Therefore, it is worthy to discover and 
analyze biomarkers for prognosis prediction of UCEC. LncRNAs have increasingly seized the attention of cancer research fields because of serving as regulating biomarkers[23]. But due to experimental complexity, functional studies related to IncRNAs have limitations to carry out in comparison with those of protein-encoding RNAs. As is illustrated in accumulating researches, molecular mechanisms underlying ceRNA network provide an explanation for carcinogenesis and its associated development. LncRNAs act as key components of ceRNA family, through miRNA response elements (MREs), compete with molecules binding to the same miRNAs to achieve regulation of expression levels between each other.

As our IncRNA-mediated ceRNA network of UCEC respectively constructed from the CPTAC and TCGA databases indicated, there were a total of 23 IncRNAs, 9 miRNAs, and 33 mRNAs correlated with the overall survival results and served as promising biomarkers for predicting prognosis of UCEC. Conventional prognostic model constructions often make inadequate risk groupings and estimates of clinical outcomes[24, 25]. Nonetheless, this ceRNA hypothesis provides us a novel predictive insight from the angle of heterogeneity between UCEC patients to analyze overall survival (OS) results. For bioinformatics analysis conducted in multiple databases, it is common practice to combine sample profiles from multiple databases for further analysis after standardized quality control treatment. However, the follow-up time length of UCEC profiles from CPTAC is shorter than that from TCGA database. So we calculated on the level of three-year survival results thus didn't combine it with that from TCGA database.

In our present study, MIR205HG and ADARB2-AS1 were significantly correlated with survival. High expression of MIR205HG was found at the first time to predict a good prognosis, while high expression of ADARB2-AS1 had an opposite effect on the survival outcome of patients. Dong et al. showed that IncRNA MIR205HG depleted SRSF1 to increase KRT17 expression[26], while KRT17 silencing impaired cervical cancer cell proliferation and migration and activated apoptosis. LncRNA MIR205HG also acts as a ceRNA to accelerate tumor growth and progression in cervical cancer through spongiform Mir-122-5p[27]. ADARB2-AS1 has been reported as a prognostic related IncRNA in UCEC[28], which again echoed the reliability of our results.

In addition, in order to make our results convincing, we put IncRNA biomarkers into GEPIA for external verification. The results showed that 5 highly expressed IncRNAs of DGCR5, GLIS3-AS1, UPK1A-AS1, MEG8, TPTEP1 indicated poor prognosis, which is in agreement with our results. To clarify our findings, we comprehensively analyzed survival results of IncRNA-regulated mRNAs in GEPIA and HPA. Through these external databases, we also concluded that high expression of CCL22, CCNE1, IGFBP5, NR3C1 and POLQ genes were associated with poor prognosis of UCEC. Furthermore, we mirrored methods in foregoing study[29] to identify our mRNA results and obtained poor survival results of NR3C1 and CCNE1 by validations of IHC images, which suggested their tumor promoter roles. Some researches has verified that CCNE1 amplification is associated with aggressive potential in UCEC tumorigenesis[30-32]. CCNE1, known as Cyclin E1, is a member of Cyclins to function as regulators of CDK kinases. The protein encoded by Cyclin belongs to the highly conserved Cyclin family, characterized in its dramatic periodicity 
in protein abundance through cell cycle. With respect to other carcinoma progression, patients with overexpressed CCNE1 were reportedly at increased threat for poor endings of cervical cancer[33] and triplenegative breast cancer[34]. Functioned as regulatory genes in the downstream, mRNA CCNE1 and NR3C1 brought potential reference values for our presently identified IncRNA-mediated ceRNA pathways.

Furthermore, on the basis of overall ceRNA network, we constructed a novel prognostic ceRNA subnetwork, which were composed of IncRNA-miRNA-mRNA axes. We firstly conducted survival analysis and multivariate analysis on IncRNAs in the ceRNA network derived from TCGA database, and identified them as hub genes in the following subnetwork. We firstly identified these key IncRNAs in the ceRNA network of TCGA, then paired their corresponding key miRNAs. Then we matched the key miRNAs with survival-related mRNAs thus to construct the survival-related subnetwork. For survival analysis in CPTAC database, although we failed to identify survival-related mRNAs and miRNAs, we surprisingly discovered that survival-related mRNAs and miRNAs in TCGA database also existed in the ceRNA network constructed by CPTAC database. Therefore, we chose to use these mRNAs, miRNAs and key IncRNAs derived from CPTAC to jointly construct survival-related subnetworks of CPTAC. In our constructed ceRNA subnetwork, there were 6 IncRNA-mediated IncRNA-miRNA-mRNA axes to role in survival outcomes of UCEC patients. A novel survival-related InCRNA DGCR5 could up-regulate CCNE1 expression by binding to miR195, miR383 and miR424, although DGCR5 had not been directly recorded in UCEC tumorigenesis procedures. DiGeorge syndrome critical region gene 5 (DGCR5), a molecular sponge to regulate cancerous signaling pathways, has been previously discovered to be extremely dysregulated in various tumors and induce the malignant phenotypes of oragans such as liver, pancreas and lungs.etc. Except for DGCR5, IncRNA LINC00443, LINC00483, C2orf48, TRBV11-2 and MEG8 were identified by multivariate Cox regression analysis, which reveals more accurate ability to predict prognosis.

From multivariate Cox regression analysis in our constructed ceRNA network, we totally identified 10 InCRNA prognostic signature candidates to predict the survival events of UCEC patients. As shown in our ceRNA subnetwork diagram for TCGA, IncRNAs such as LINC00443, C2orf48, LINC00483 could regulate DLX4 and NR3C1 expression by binding to miR-183. LINC00443, LINC00483 and C2orf48 were previously proved to promote carcinoma progression[35], the same consequence of which were validated by our experiment. DLX4 has been shown to cause tumor migration, invasion, and metastasis[36]. Previous in vivo studies on UCEC reported that DLX4 promoted cell proliferation, migration, and suggested poor prognosis, which is consistent with our findings[37]. NR3C1 encodes glucocorticoid receptors to affect glucocorticoid response and participates in other transcription regulatory procedures. Former study on miRNA-mRNA regulatory network in UCEC found that over expression of mRNA NR3C1 led to poor prognosis[38, 39]. Previously in vivo experiments proved that over-expressed LINC00483 promoted UCEC tumorigenesis, the mechanism of which was mainly to sponge with miR-508-3p to regulate RGS17 expression levels[40]. In other cancer researches, LINC00483 also acted as a strong ceRNA molecule and exhibited its regulatory ability to mediate tumor progression and prognosis, such as lung adenocarcinoma[41] and gastric cancer[42]. Our clinical survival and transcriptome results revealed that patients with over-expression of LINC00443, C2orf48 and LINC00483 had poor prognostic outcomes. The two IncRNAs filtered in multi-Cox regression analysis in CPTAC, TRBV11-2 and MEG8 provided potential 
pathways to explain ceRNA regulatory network despite of none associated reports for UCEC. Firstly, they could up-regulate SOX11 expression by binding to hsa-mir-363 to bring about poor prognosis, and survival related mRNA SOX11 hypermethylation was reported as a tumor biomarker in UCEC[43]. Secondly, MEG8 competed with has-mir-424 to CBX2 and CCNE1 expression as well as competing with has-mir-183 to up-regulate DLX4 and NR3C1 expression. Therefore, we predicted that TRBV11-2 and MEG8 worked as prognostic ceRNAs to up-regulate SOX11, CCNE1 and NR3C1 expression thus resulting in poor prognosis, suggesting that these IncRNAs could promote UCEC development. Furthermore, LINC00028 existed in our survival analysis results rooted from both databases, and the over-expression of LINC00028 indicated a poor prognosis. Besides, LINC00028 has been reported to be involved in ceRNA regulatory network of osteosarcoma recurrence[44]. However, its mechanisms related to UCEC pathogenesis remains unclear.

As illustrated in former studies[45, 46], UCEC carcinogenesis is promoted by cell cycle acceleration. Similarly, our KEGG analyses in both databases indicated that DEmRNAs were mostly enriched in pathways such as microRNAs in cancer, cellular senescence and proteoglycans in cancer.

Deoxyribonucle, and deoxyRNA were identified in tumor and adjacent normal tissue samples in a large cohort of UCEC patients.

Even though we identified the molecular mechanism of ceRNA from 587 samples in TCGA and 116 samples in CPTAC database, there was still a limited sample size for more reliable biomarkers that hindered us from incorporating data profiles originated from CPTAC and TCGA database into a comprehensive study ideally. Concerning the pilot study limited by failure to closely link the analysis of the two databases, multicentric studies are supposed to carry out to support our new researches inevitably.

\section{Conclusion}

Using data obtained from CPTAC and TCGA, we screened out IncRNA prognostic signatures on the basis of ceRNA completely composed of hub genes. Besides, an IncRNA-mediated ceRNA network reveals the molecular mechanism that facilitates UCEC pathological progress. LncRNAs including DGCR5, LINC00443, C2orf48, LINC00483, TRBV11-2 and MEG8 involved in IncRNA-miRNA-mRNA regulatory network were identified as promising diagnostic, therapeutic or prognostic biomarkers. Further studies are warranted to explore meaningful biological functional pathways underlying these IncRNA roles for UCEC.

\section{Abbreviations}

ceRNA:competing endogenous RNA; DEGs: Differentially expressed genes;IncRNA:Iong noncoding RNA;mRNA:messenger RNA; miRNA :microRNA;UCEC:uterine corpus endometrial carcinoma;TIIC:tumor infiltrating immune cell;TCGA:The Cancer Genome Atlas; CPTAC:Clinical Proteomic Tumor Analysis Consortium;STRING:Search Tool for the Retrieval of Interacting Genes; HPA:Human Protein Atlas Portal;ssGSEA:single sample gene set enrichment analysis;IHC:Immunohistonchemistry; GEPIA: Gene 
Expression Profiling Interactive Analysis;GO: Gene ontology; KEGG: Kyoto encyclopedia of genes and genomes; PPI: Protein-protein interact

\section{Declarations}

\section{Ethics approval and consent to participate}

Not applicable.

TCGA and CPTAC belong to public databases. The patients involved in the database have obtained ethical approval. Users can download relevant data for free for research and publish relevant articles. Our study is actually an integrated bioinformatics analysis based on open source data, so there are no ethical issues and other conflicts of interest. It is specially noted that the Immunohistonchemistry images mentioned in the article are retrieved and downloaded from the CPTAC website requiring no additional ethical approval.

\section{Consent for publication}

Not applicable.

\section{Availability of data and materials}

In our study, different web-based datasets were used for our further data analysis. The web links to all the original data sources were listed as below: The datasets including the RNA-seq transcriptome data and clinical data of UCEC cohort were respectively obtained from The Cancer Genome Atlas Program (TCGA) (https://portal.gdc.cancer.gov/) and National Cancer Institute Clinical Proteomic Tumor Analysis Consortium (https://cptac-data-portal.georgetown.edu/) data portal. All data generated from the analysis process of this study are available from authors listed above on reasonable request.

\section{Competing interests}

The authors declare that they have no competing interests.

\section{Funding}

This project was supported by the grant from Excellent Key Teachers in the "Qing Lan Project" of Jiangsu Colleges and Universities and "226 Project" of Nantong[(2018)III-436]. The funding body listed above played no role in the design of the study, collection, analysis, or interpretation of data, nor was it involved in the preparation of this manuscript.

\section{Author s' contributions}

YRC prepared the dataset and analyzed the data. JC wrote and revised the manuscript. HQW reviewed, modified and provided experimental conditional support. All authors have read, revised, and approved the 
final manuscript.

\section{Acknowledgements}

Not applicable.

\section{Authors' information}

${ }^{1}$ Medical School of Nantong University, Nantong, 226001, China.

${ }^{2}$ Department of Medical Informatics, Medical School of Nantong University, Nantong, 226001, China.

\section{References}

1. Calle EE, Kaaks R: Overweight, obesity and cancer: epidemiological evidence and proposed mechanisms. Nat Rev Cancer 2004, 4(8):579-591.

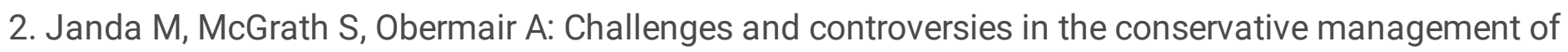
uterine and ovarian cancer. Best Pract Res Clin Obstet Gynaecol 2019, 55:93-108.

3. Lu KH, Broaddus RR: Endometrial Cancer. N Engl J Med 2020, 383(21):2053-2064.

4. Engelsen IB, Stefansson I, Akslen LA, Salvesen HB: Pathologic expression of p53 or p16 in preoperative curettage specimens identifies high-risk endometrial carcinomas. Am J Obstet Gynecol 2006, 195(4):979-986.

5. Smith D, Stewart CJR, Clarke EM, Lose F, Davies C, Armes J, Obermair A, Brennan D, Webb PM, Nagle $\mathrm{CM}$ et al: ER and PR expression and survival after endometrial cancer. Gynecol Oncol 2018, 148(2):258-266.

6. Wright JD, Burke WM, Wilde ET, Lewin SN, Charles AS, Kim JH, Goldman N, Neugut Al, Herzog TJ, Hershman DL: Comparative effectiveness of robotic versus laparoscopic hysterectomy for endometrial cancer. J Clin Oncol 2012, 30(8):783-791.

7. Bartel DP: MicroRNAs: genomics, biogenesis, mechanism, and function. Cell 2004, 116(2):281-297.

8. Peng Y, Croce CM: The role of MicroRNAs in human cancer. Signal Transduct Target Ther 2016, $1: 15004$.

9. Zhang Z, Gu M, Gu Z, Lou YR: Role of Long Non-Coding RNA Polymorphisms in Cancer Chemotherapeutic Response. J Pers Med 2021, 11(6).

10. Schmitt AM, Chang HY: Long Noncoding RNAs in Cancer Pathways. Cancer Cell 2016, 29(4):452463.

11. Ahadi A: Functional roles of IncRNAs in the pathogenesis and progression of cancer. Genes Dis 2021, 8(4):424-437.

12. Salmena L, Poliseno L, Tay Y, Kats L, Pandolfi PP: A ceRNA hypothesis: the Rosetta Stone of a hidden RNA language? Cell 2011, 146(3):353-358. 
13. Yang J, Qiu Q, Qian X, Yi J, Jiao Y, Yu M, Li X, Li J, Mi C, Zhang J et al: Long noncoding RNA LCAT1 functions as a ceRNA to regulate RAC1 function by sponging miR-4715-5p in lung cancer. Mol Cancer 2019, 18(1):171.

14. Sun $\mathrm{M}$, Gomes $\mathrm{S}$, Chen P, Frankenberger CA, Sankarasharma D, Chung $\mathrm{CH}$, Chada KK, Rosner MR: RKIP and HMGA2 regulate breast tumor survival and metastasis through lysyl oxidase and syndecan-2. Oncogene 2014, 33(27):3528-3537.

15. Franceschini A, Szklarczyk D, Frankild S, Kuhn M, Simonovic M, Roth A, Lin J, Minguez P, Bork P, von Mering $C$ et al: STRING v9.1: protein-protein interaction networks, with increased coverage and integration. Nucleic Acids Res 2013, 41(Database issue):D808-815.

16. Smoot ME, Ono K, Ruscheinski J, Wang PL, Ideker T: Cytoscape 2.8: new features for data integration and network visualization. Bioinformatics 2011, 27(3):431-432.

17. Chin $\mathrm{CH}$, Chen $\mathrm{SH}, \mathrm{Wu} \mathrm{HH}, \mathrm{Ho} \mathrm{CW}$, Ko MT, Lin CY: cytoHubba: identifying hub objects and subnetworks from complex interactome. BMC Syst Biol 2014, 8 Suppl 4(Suppl 4):S11.

18. Huang HY, Lin YC, Li J, Huang KY, Shrestha S, Hong HC, Tang Y, Chen YG, Jin CN, Yu Y et al: miRTarBase 2020: updates to the experimentally validated microRNA-target interaction database. Nucleic Acids Res 2020, 48(D1):D148-d154.

19. Wong N, Wang X: miRDB: an online resource for microRNA target prediction and functional annotations. Nucleic Acids Res 2015, 43(Database issue):D146-152.

20. Fromm B, Billipp T, Peck LE, Johansen M, Tarver JE, King BL, Newcomb JM, Sempere LF, Flatmark K, Hovig E et al: A Uniform System for the Annotation of Vertebrate microRNA Genes and the Evolution of the Human microRNAome. Annu Rev Genet 2015, 49:213-242.

21. Uhlén M, Fagerberg L, Hallström BM, Lindskog C, Oksvold P, Mardinoglu A, Sivertsson Å, Kampf C, Sjöstedt E, Asplund A et al: Proteomics. Tissue-based map of the human proteome. Science 2015, 347(6220):1260419.

22. Siegel RL, Miller KD, Jemal A: Cancer statistics, 2019. CA Cancer J Clin 2019, 69(1):7-34.

23. Shi X, Sun M, Liu H, Yao Y, Song Y: Long non-coding RNAs: a new frontier in the study of human diseases. Cancer Lett 2013, 339(2):159-166.

24. Huo $X$, Sun $H$, Cao D, Yang J, Peng P, Yu M, Shen K: Identification of prognosis markers for endometrial cancer by integrated analysis of DNA methylation and RNA-Seq data. Sci Rep 2019, 9(1):9924.

25. Ouyang D, Li R, Li Y, Zhu X: A 7-IncRNA signature predict prognosis of Uterine corpus endometrial carcinoma. J Cell Biochem 2019, 120(10):18465-18477.

26. Dong M, Dong Z, Zhu X, Zhang Y, Song L: Long non-coding RNA MIR205HG regulates KRT17 and tumor processes in cervical cancer via interaction with SRSF1. Exp Mol Pathol 2019, 111:104322.

27. Li Y, Wang H, Huang H: Long non-coding RNA MIR205HG function as a ceRNA to accelerate tumor growth and progression via sponging miR-122-5p in cervical cancer. Biochem Biophys Res Commun 2019, 514(1):78-85. 
28. Xia L, Wang Y, Meng Q, Su X, Shen J, Wang J, He H, Wen B, Zhang C, Xu M: Integrated Bioinformatic Analysis of a Competing Endogenous RNA Network Reveals a Prognostic Signature in Endometrial Cancer. Front Oncol 2019, 9:448.

29. Zhang X, Feng H, Li Z, Li D, Liu S, Huang H, Li M: Application of weighted gene co-expression network analysis to identify key modules and hub genes in oral squamous cell carcinoma tumorigenesis. Onco Targets Ther 2018, 11:6001-6021.

30. Kuhn E, Bahadirli-Talbott A, Shih le M: Frequent CCNE1 amplification in endometrial intraepithelial carcinoma and uterine serous carcinoma. Mod Pathol 2014, 27(7):1014-1019.

31. Leskela S, Pérez-Mies B, Rosa-Rosa JM, Cristobal E, Biscuola M, Palacios-Berraquero ML, Ong S, Matias-Guiu Guia X, Palacios J: Molecular Basis of Tumor Heterogeneity in Endometrial Carcinosarcoma. Cancers (Basel) 2019, 11(7).

32. Nakayama K, Rahman MT, Rahman M, Nakamura K, Ishikawa M, Katagiri H, Sato E, Ishibashi T, lida $\mathrm{K}$, Ishikawa $\mathrm{N}$ et al: CCNE1 amplification is associated with aggressive potential in endometrioid endometrial carcinomas. Int J Oncol 2016, 48(2):506-516.

33. Zhang Y, Li X, Zhang J, Mao L: E6 hijacks KDM5C/Inc_000231/miR-497-5p/CCNE1 axis to promote cervical cancer progression. J Cell Mol Med 2020, 24(19):11422-11433.

34. Yang R, Xing L, Zheng X, Sun Y, Wang X, Chen J: The circRNA circAGFG1 acts as a sponge of miR195-5p to promote triple-negative breast cancer progression through regulating CCNE1 expression. Mol Cancer 2019, 18(1):4.

35. Liu J, Nie S, Liang J, Jiang Y, Wan Y, Zhou S, Cheng W: Competing endogenous RNA network of endometrial carcinoma: A comprehensive analysis. J Cell Biochem 2019, 120(9):15648-15660.

36. Zhang L, Yang M, Gan L, He T, Xiao X, Stewart MD, Liu X, Yang L, Zhang T, Zhao Y et al: DLX4 upregulates TWIST and enhances tumor migration, invasion and metastasis. Int J Biol Sci 2012, 8(8):1178-1187.

37. Zhang L, Wan Y, Jiang Y, Zhang Z, Shu S, Cheng W, Lang J: Overexpression of BP1, an isoform of Homeobox Gene DLX4, promotes cell proliferation, migration and predicts poor prognosis in endometrial cancer. Gene 2019, 707:216-223.

38. Ding H, Fan GL, Yi YX, Zhang W, Xiong XX, Mahgoub OK: Prognostic Implications of Immune-Related Genes' (IRGs) Signature Models in Cervical Cancer and Endometrial Cancer. Front Genet 2020, $11: 725$.

39. Sun R, Liu J, Nie S, Li S, Yang J, Jiang Y, Cheng W: Construction of miRNA-mRNA Regulatory Network and Prognostic Signature in Endometrial Cancer. Onco Targets Ther 2021, 14:2363-2378.

40. Hu P, Zhou G, Zhang X, Song G, Zhan L, Cao Y: Long non-coding RNA Linc00483 accelerated tumorigenesis of cervical cancer by regulating miR-508-3p/RGS17 axis. Life Sci 2019, 234:116789.

41. Yang S, Liu T, Sun Y, Liang X: The long noncoding RNA LINC00483 promotes lung adenocarcinoma progression by sponging miR-204-3p. Cell Mol Biol Lett 2019, 24:70.

42. Li D, Yang M, Liao A, Zeng B, Liu D, Yao Y, Hu G, Chen X, Feng Z, Du Y et al: Linc00483 as ceRNA regulates proliferation and apoptosis through activating MAPKs in gastric cancer. J Cell Mol Med 
2018, 22(8):3875-3886.

43. Shan T, Uyar DS, Wang LS, Mutch DG, Huang TH, Rader JS, Sheng X, Huang YW: SOX11 hypermethylation as a tumor biomarker in endometrial cancer. Biochimie 2019, 162:8-14.

44. Zhang S, Ding L, Li X, Fan H: Identification of biomarkers associated with the recurrence of osteosarcoma using ceRNA regulatory network analysis. Int J Mol Med 2019, 43(4):1723-1733.

45. Wang Y, Qiu H, Hu W, Li S, Yu J: RPRD1B promotes tumor growth by accelerating the cell cycle in endometrial cancer. Oncol Rep 2014, 31(3):1389-1395.

46. Xiong H, Li Q, Chen R, Liu S, Lin Q, Xiong Z, Jiang Q, Guo L: A Multi-Step miRNA-mRNA Regulatory Network Construction Approach Identifies Gene Signatures Associated with Endometrioid Endometrial Carcinoma. Genes (Basel) 2016, 7(6).

\section{Figures}


(A)

(D)

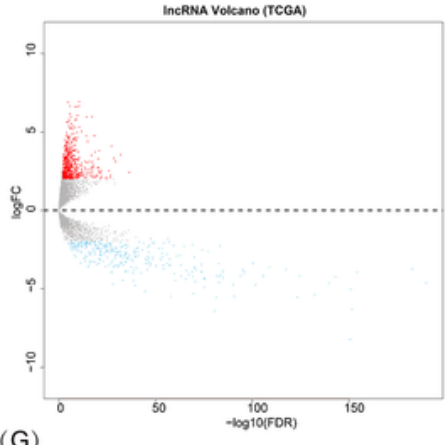

(G)

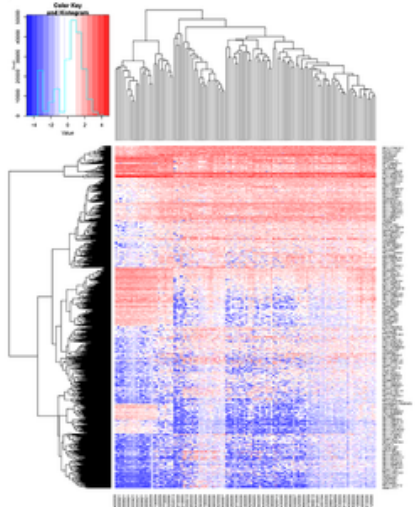

(J)

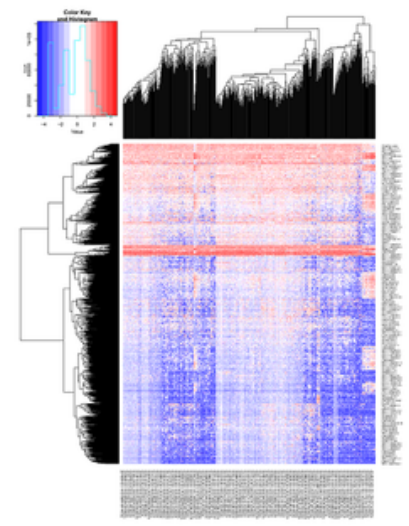

(B)

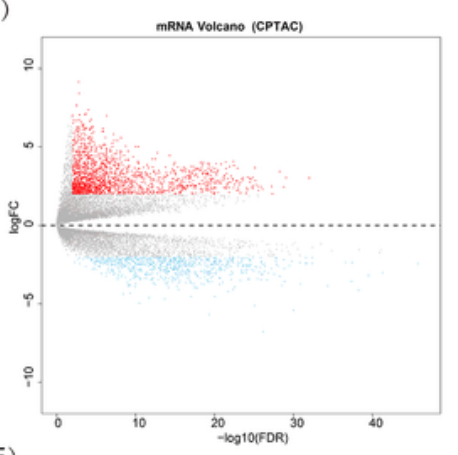

(E)

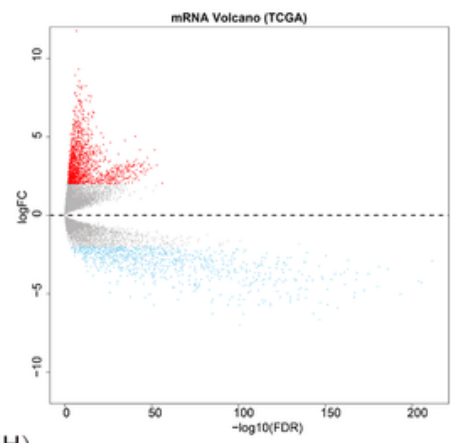

(H)

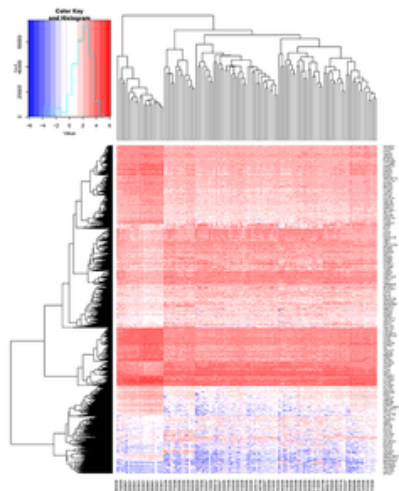

(K)

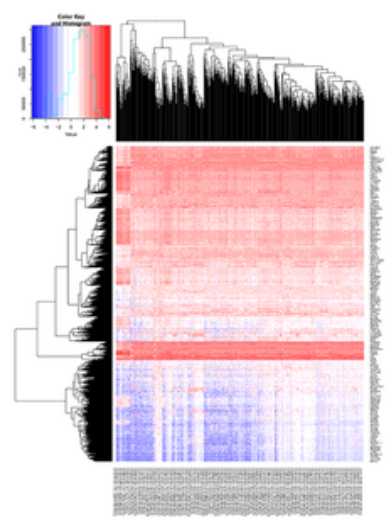

(C)

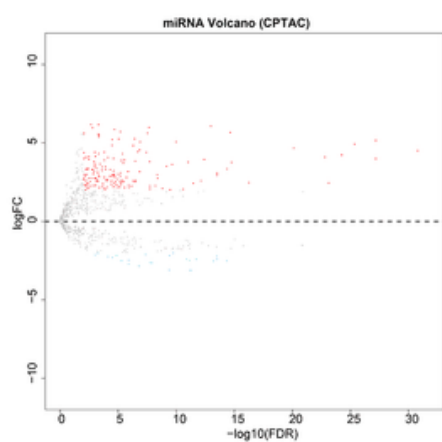

$(\mathrm{F})$

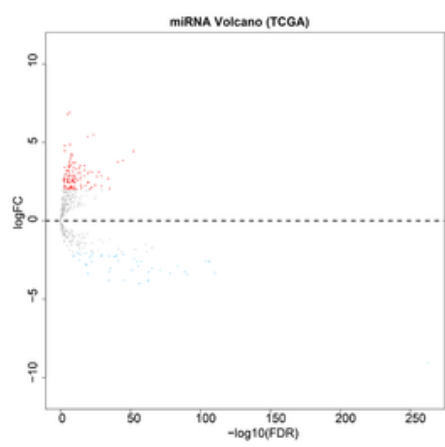

(I)

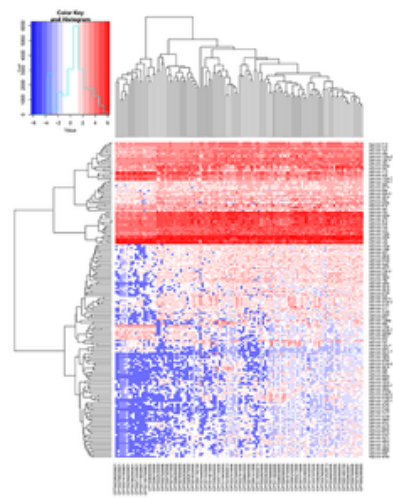

(L)

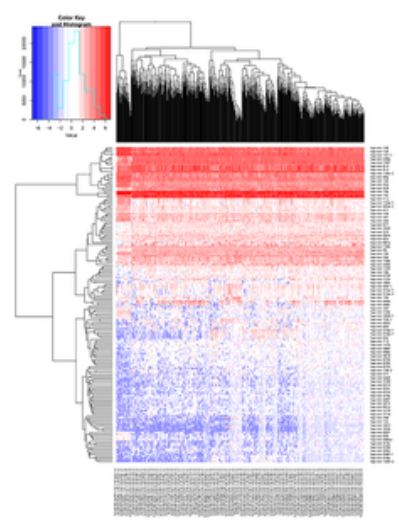

Figure 1

DERNAs between UCEC and normal tissues in the CPTAC and TCGA database. Volcano plot of UCECspecific IncRNAs (A), mRNAs (B) and miRNAs (C) from CTPAC and IncRNAs (D), mRNAs(E), miRNAs(F) from TCGA. Red dot indicated high expression, and blue dot indicated low expression. Grey showed that those genes showed no difference between cancer and non-cancer tissues. The hierarchical clustering heatmaps of DERNAs between the UCEC and normal tissues from the CPTAC(G-I) and TCGA database(J- 
L). The horizontal axis represented samples while above the horizontal axis lists sample clusters. The vertical axis represented RNAs. Red denoted up-regulated RNAs, and purple denoted down-regulated RNAs.
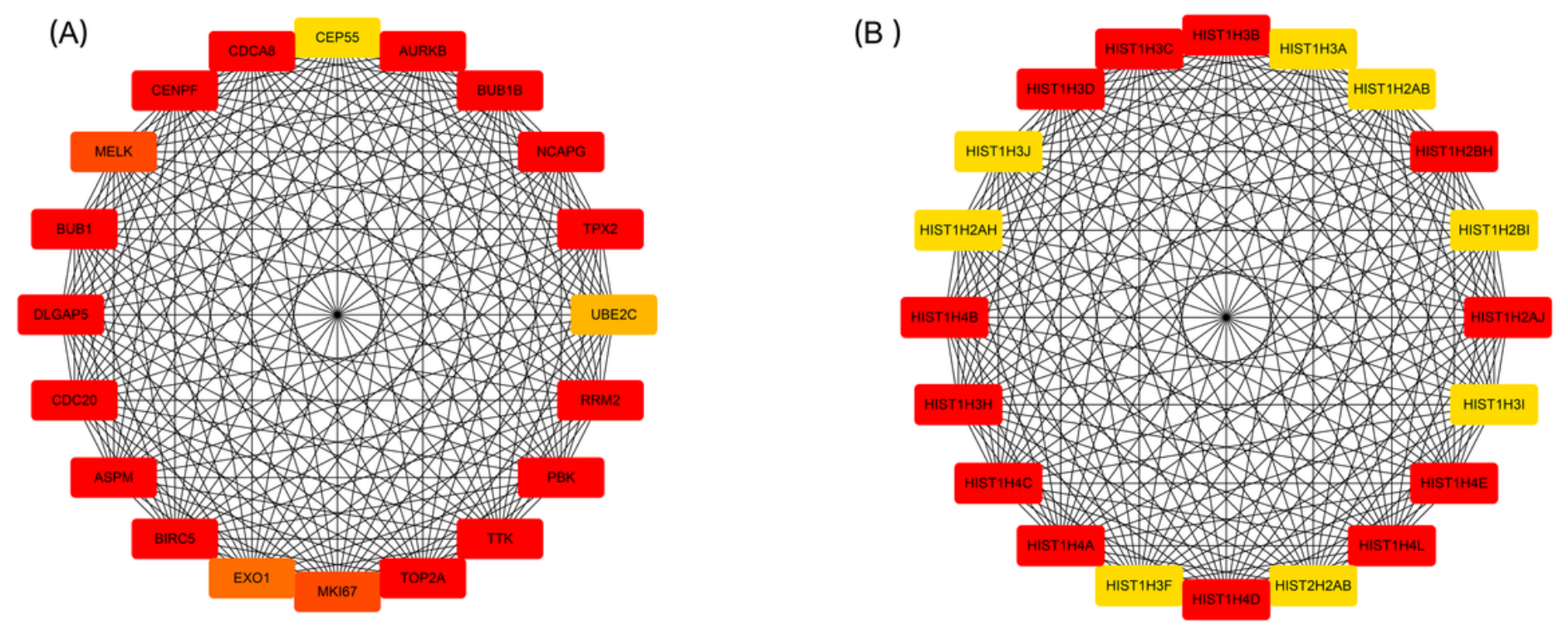

Figure 2

Constructed PPI network for DEmRNAs from CPTAC (A) and TCGA database (B). 
(A)

CPTAC

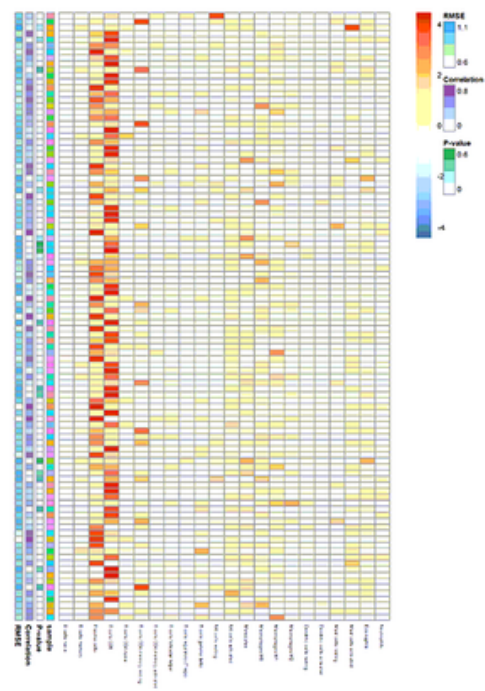

(C)

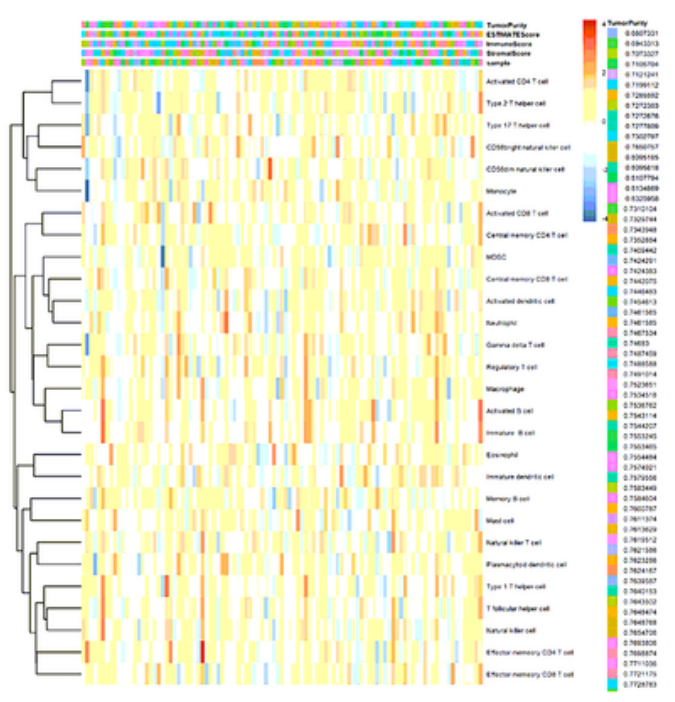

(E)

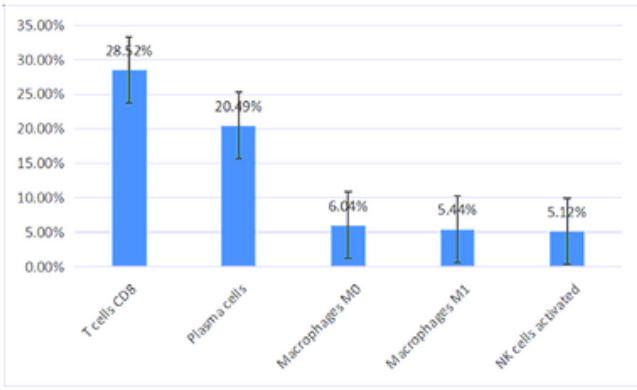

(B) TCGA

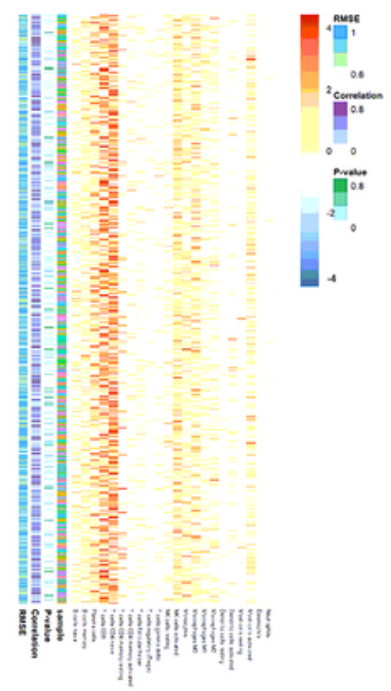

(D)

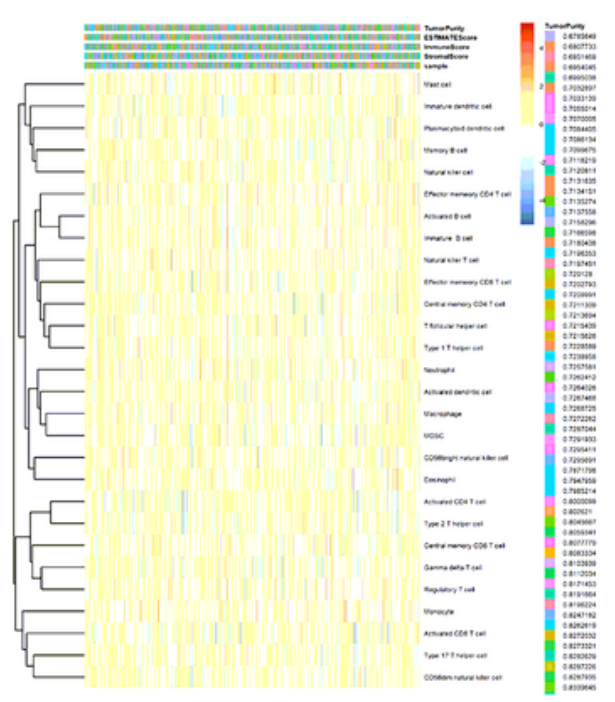

(F)

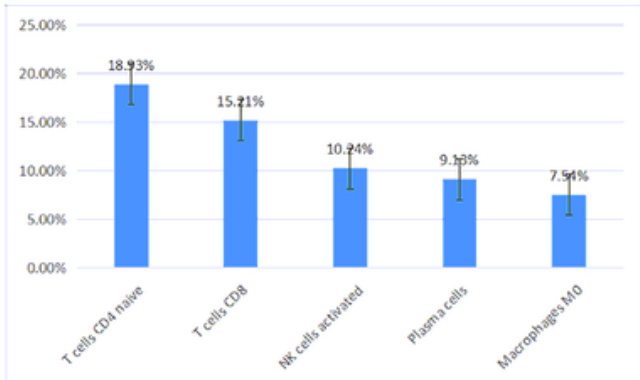

\section{Figure 3}

The immune infiltration landscape in UCEC. A, B: Estimation of 22 immune leucocytes fractions with different colors in UCEC by CIBERSORT algorithm. The vertical axis represented the cell proportions. C, D: The score of 31 immune signatures in each sample by heatmap by ssGSEA. E, F: The top five immune cell components were derived from CIBERSORT algorithm and then visualized in the bar chart. 
(A)

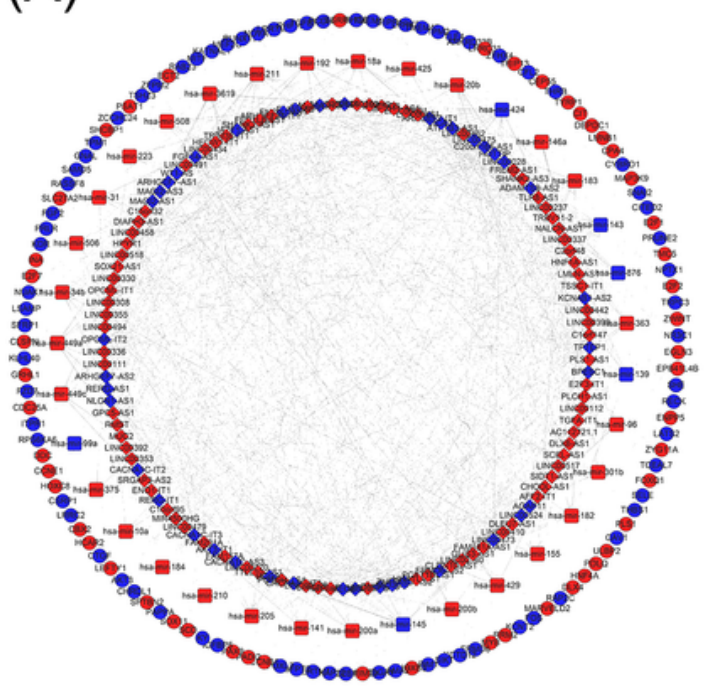

(C)

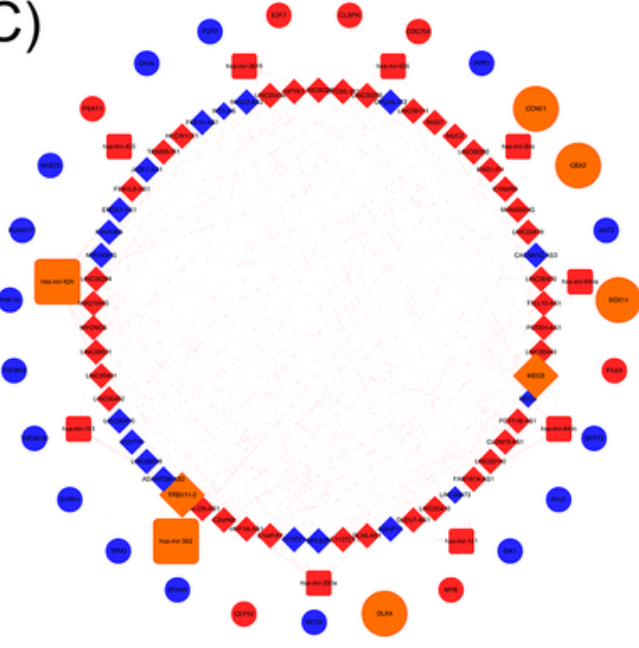

$(\mathrm{E})$

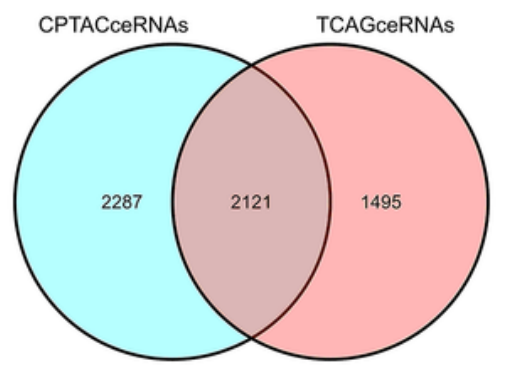

(B)

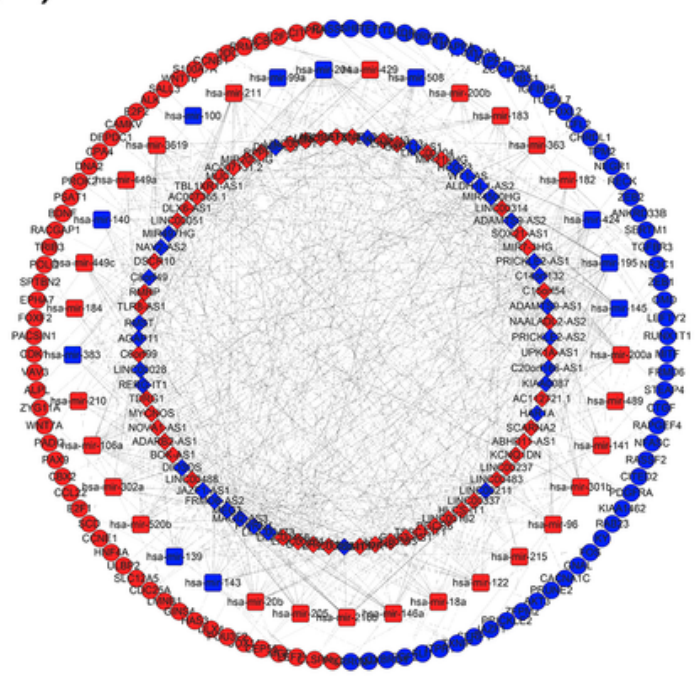

(D)

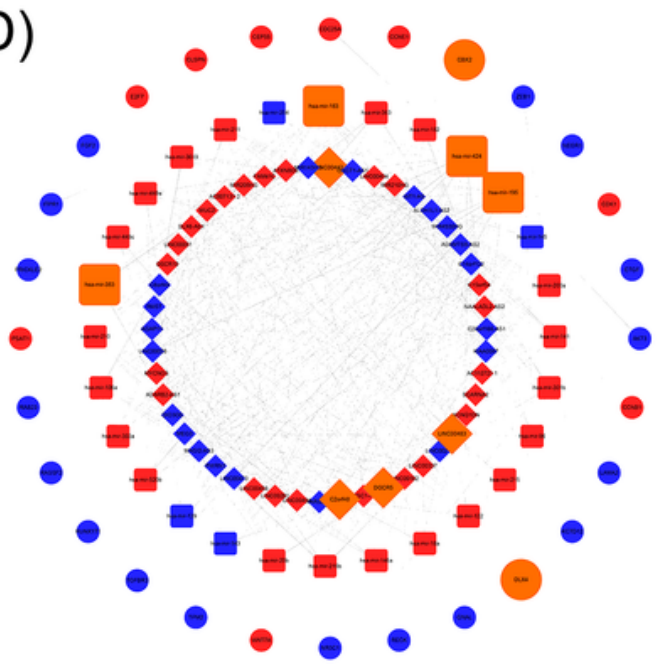

Figure 4

Overall construction of IncRNA-mediated ceRNA network in UCEC from CPTAC (A) and TCGA database (B). Diamonds, rounded rectangles and ellipses respectively represented IncRNAs, miRNAs and mRNAs. The red indicated high expression while the blue indicated low expression. Potentially prognostic ceRNA network of IncRNA-miRNA-mRNA pairs and derived regulatory genes arranged by Cytohubba from CPTAC (A) and TCGA database (B). E: Venn diagram of genes completely involved in the overall ceRNA network 
in CPTAC and TCGA database. The overlapped area in the middle indicated intersected genes of two databases.
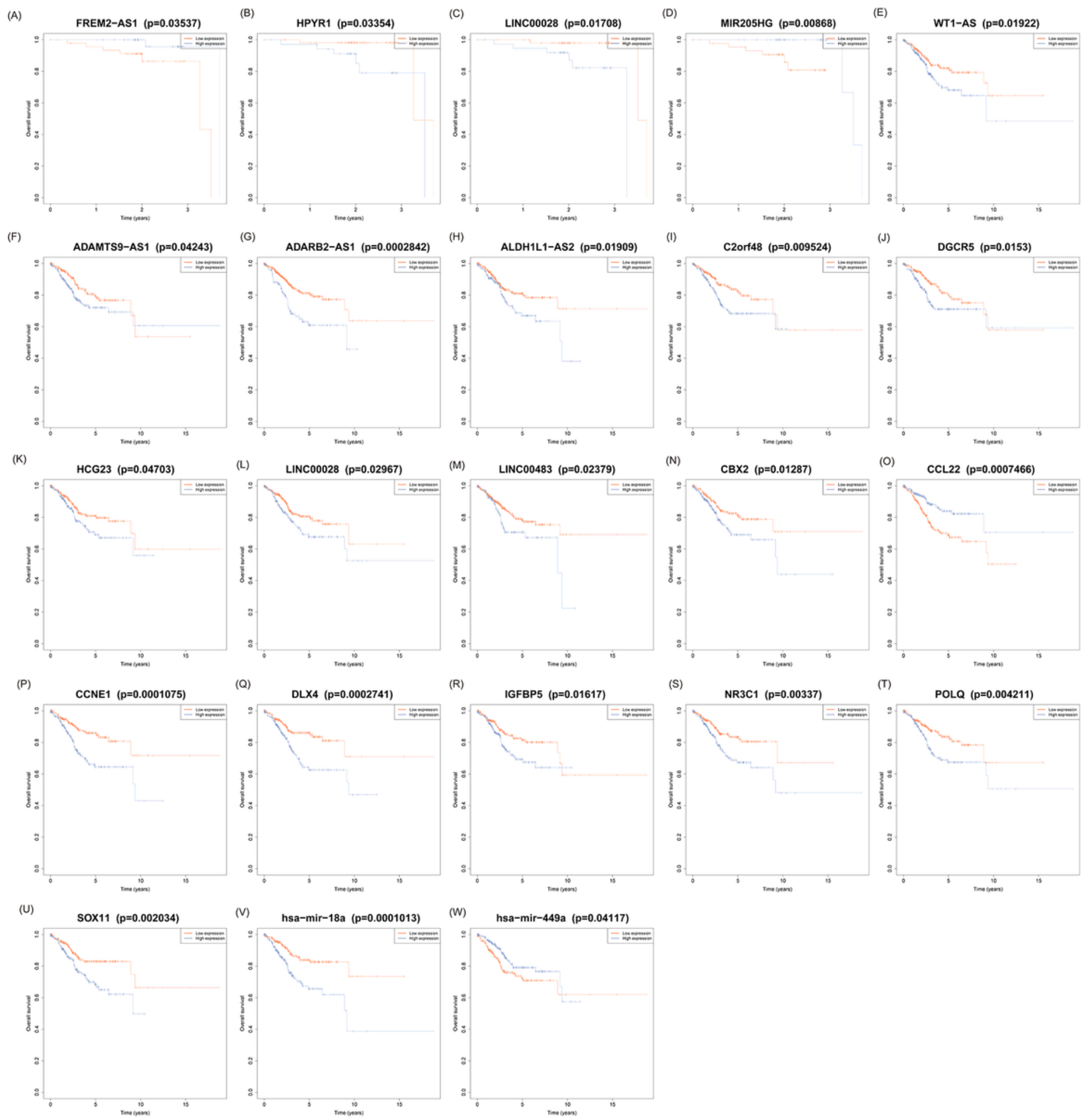

Figure 5

Kaplan-Meier curves of DERNAs and overall survival rate in UCEC samples. 
(A)

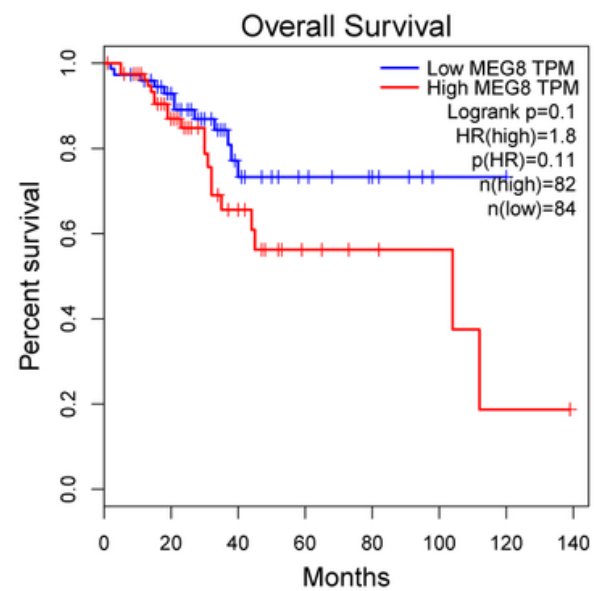

(D)

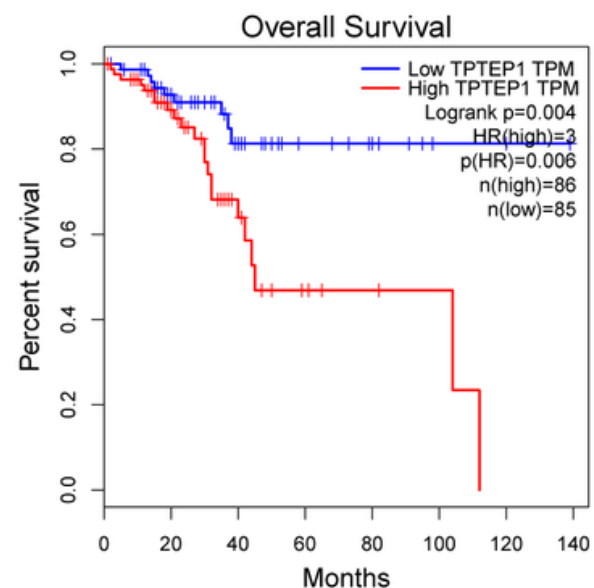

(B)

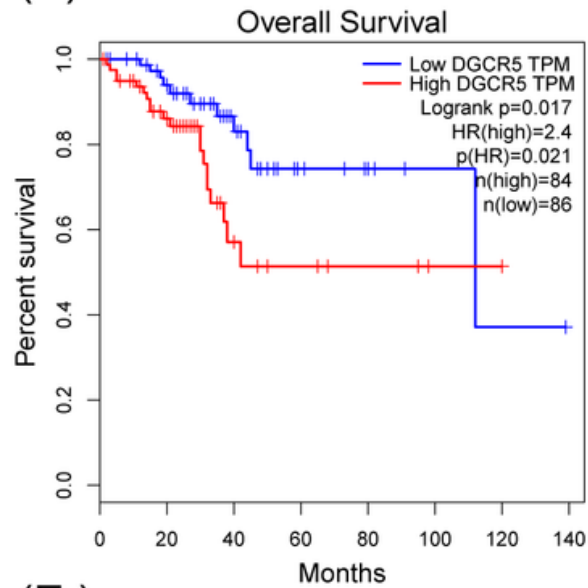

$(\mathrm{E})$

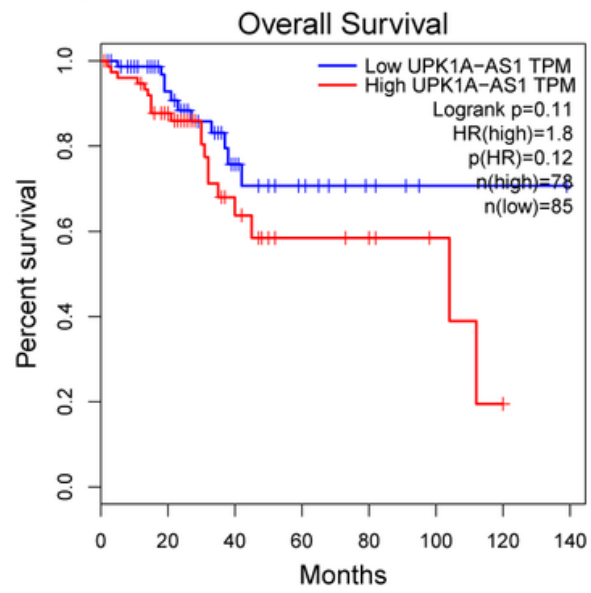

(C)

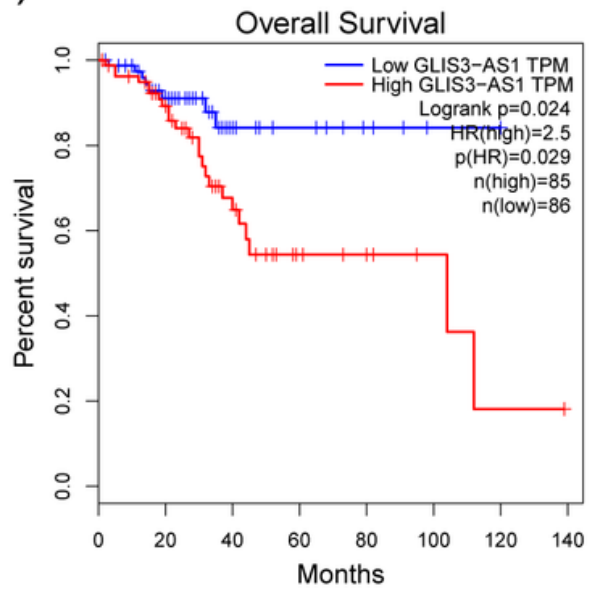

\section{Figure 6}

Kaplan-Meier curves of survival-related IncRNAs validated in GEPIA database. Graphs of IncRNAs including MEG8 (A), DGCR5 (B), GLIS3-AS1(C), TPTEP1(D), and UPK1A-AS1 (E)

(A)

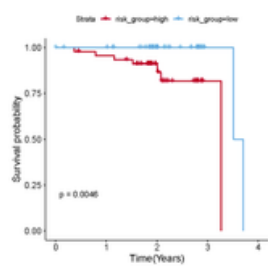

(B)

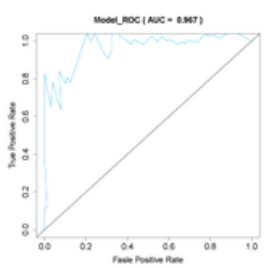

(C)

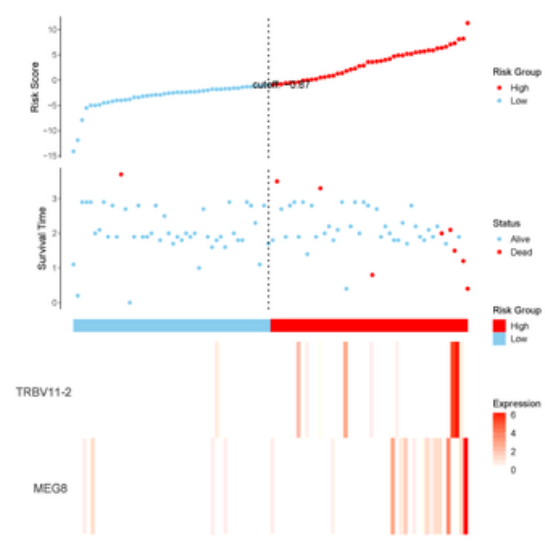

(D)

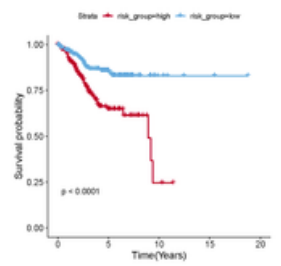

(E)

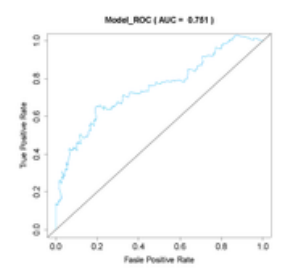

(F)

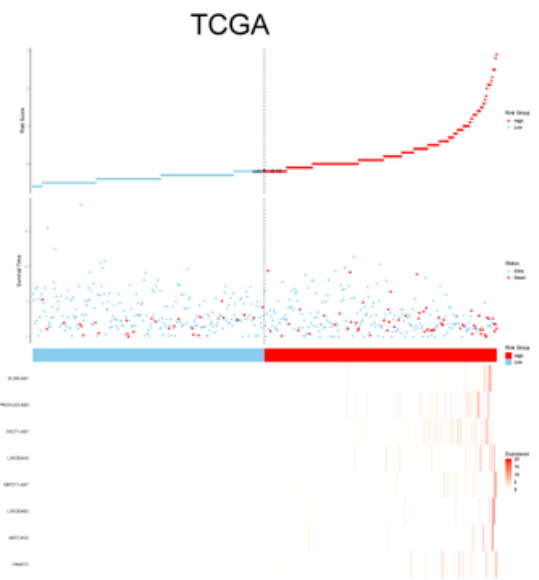

Figure 7 
LncRNA signature prognostic risk models for UCEC. For CPTAC dataset, Kaplan-Meier curves showed that low-risk group had a lower mortality rate than high-risk group $(P=0.0046<0.05)(A)$. The ROC curves of OS-related predictive signatures (AUC:0.967)(B). The high expression level of MEG8 and TRBV11_2 were significantly associated with worse OS in Kaplan-Meier curves (both $\mathrm{P}<0.05)(C)$. While for TCGA dataset, Kaplan-Meier curves showed that low-risk group had a lower mortality rate than high-risk group $(P<0.0001)(D)$. The ROC curves of OS-related predictive signatures (AUC: 0.751)(E). The high expression level of the eight IncRNAs was significantly associated with worse OS in Kaplan-Meier curves. (F)

(A)

\section{CCNE1}
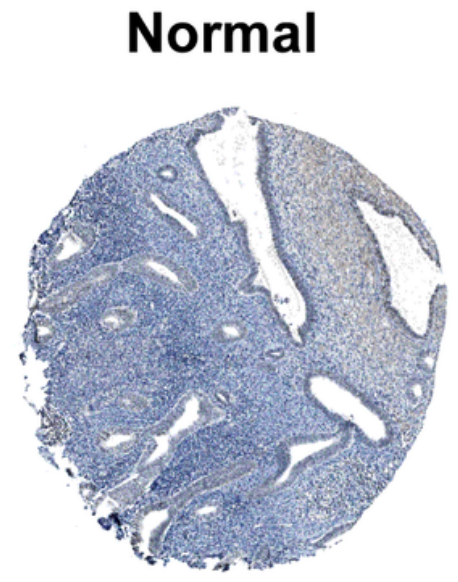

NR3C1

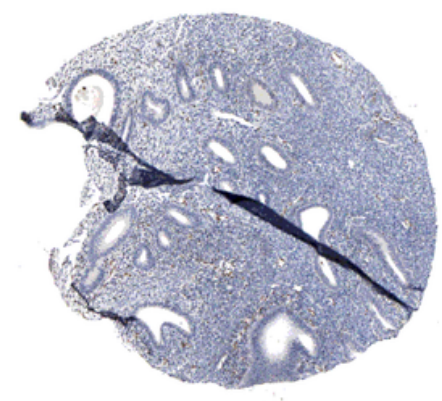

(D)

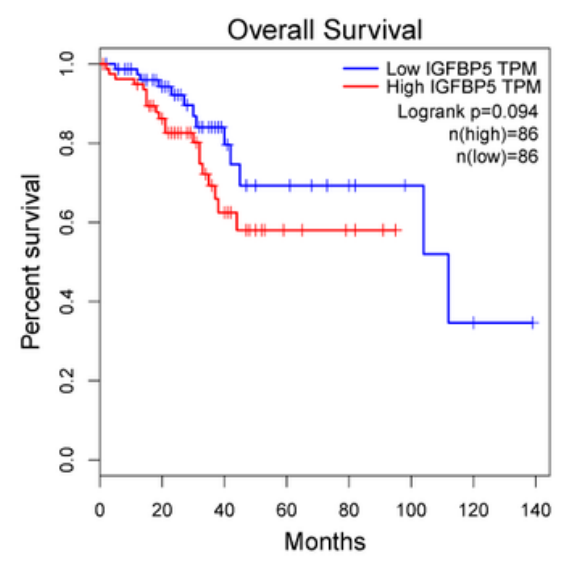

Tumor
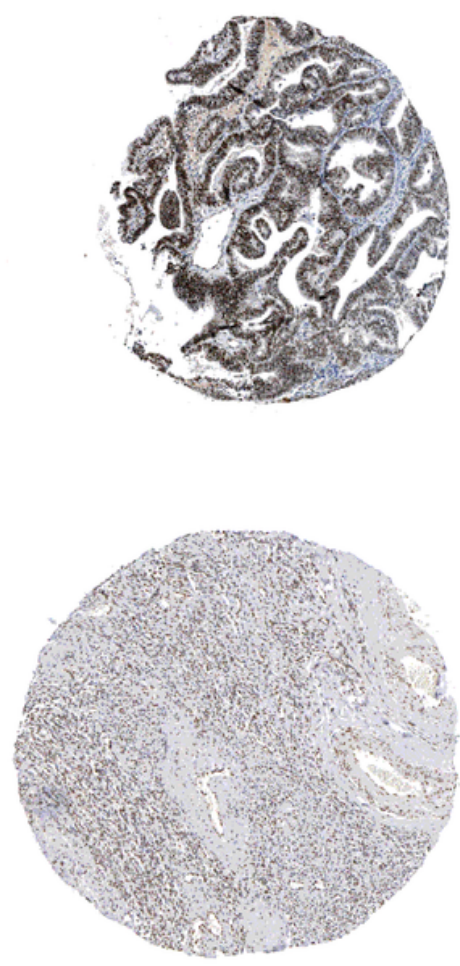

(E)

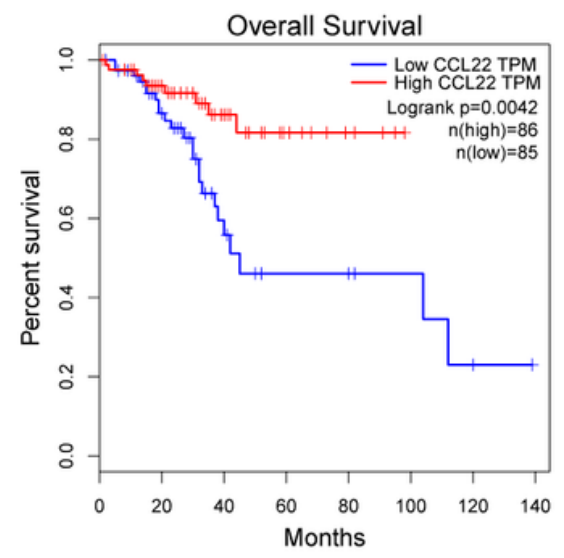

(B)

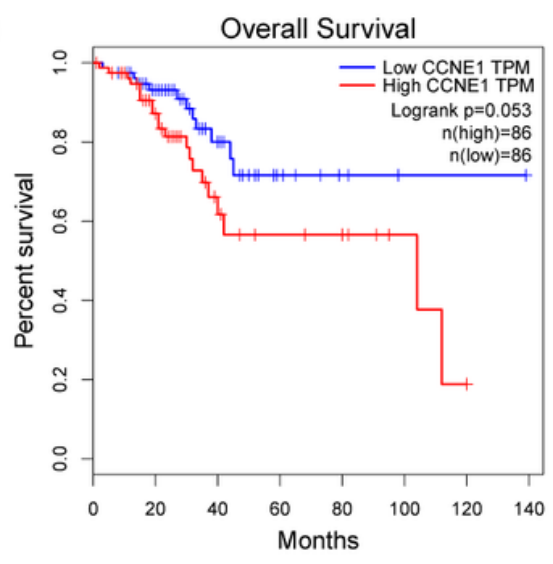

(C)

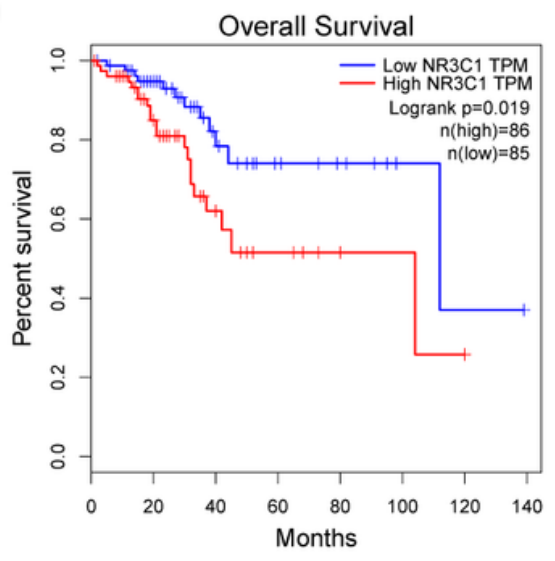

$(\mathrm{F})$

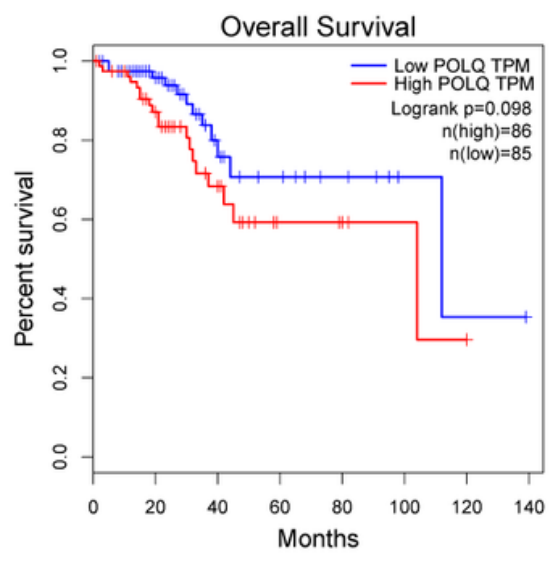

Figure 8 
Validations of survival analysis and mRNA expression at the transcriptional level. A: Validation of hub genes at the translational level by the HPA database. NR3C1 validated by antibody HPA018169. Normal human tissue id:3352; Patient tissue id:2772. CCNE1 validated by antibody HPA004248. Normal human tissue id:2102; Patient tissue id:2173. B-F: The Kaplan-Meier curves of 5 mRNAs from the HPA and GEPIA database.

(A)

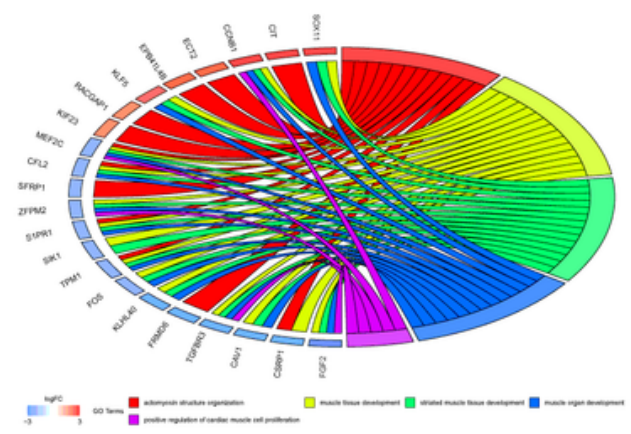

(C)

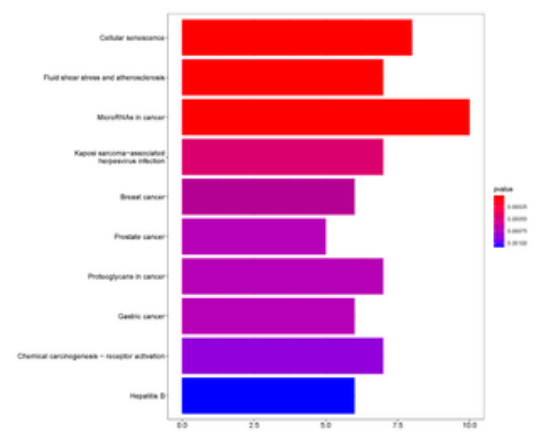

(E)

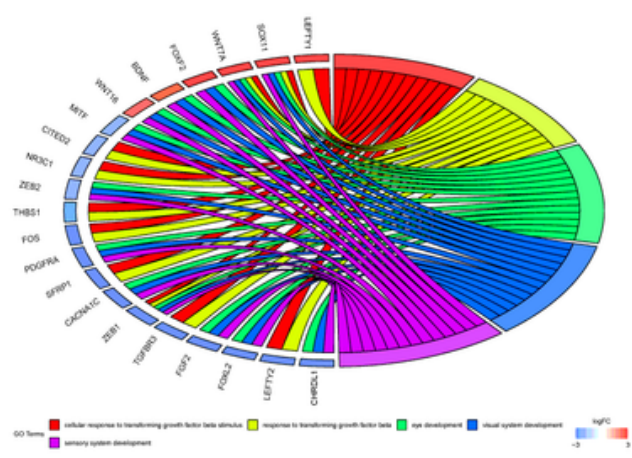

(G)

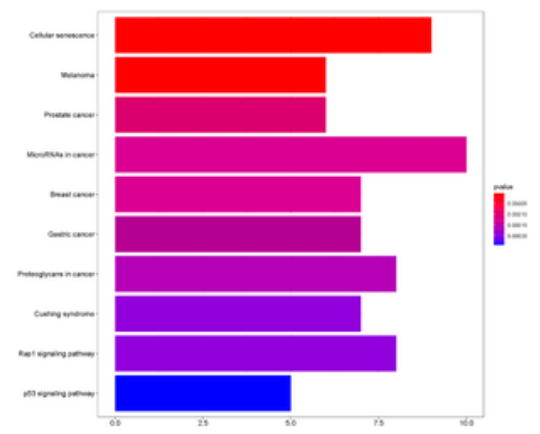

(B)

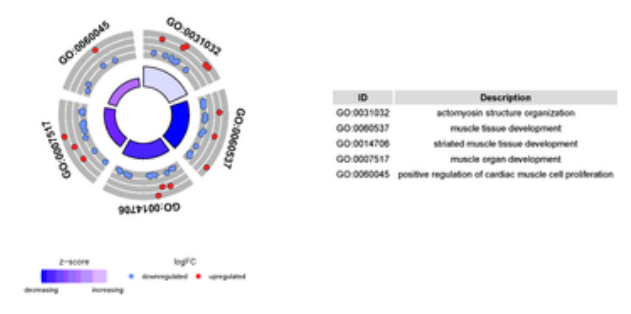

(D)

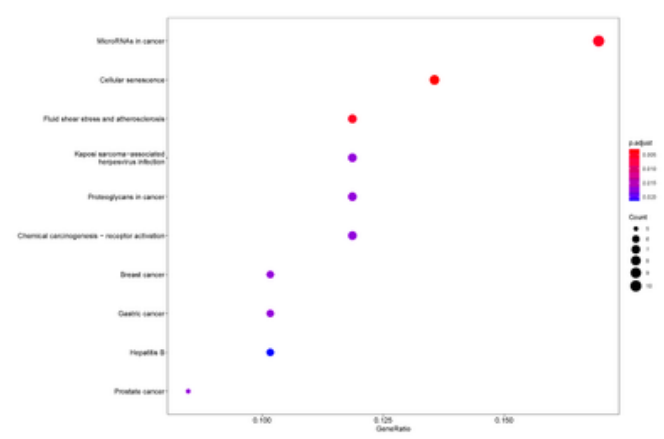

(F)

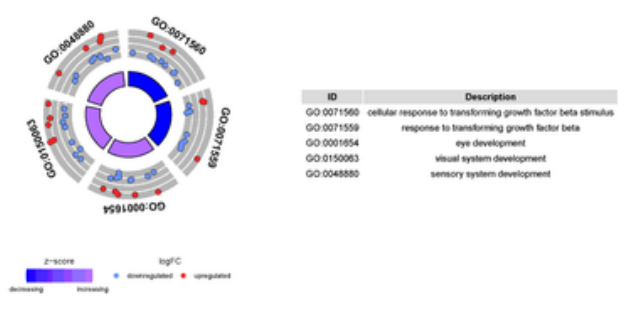

(H)

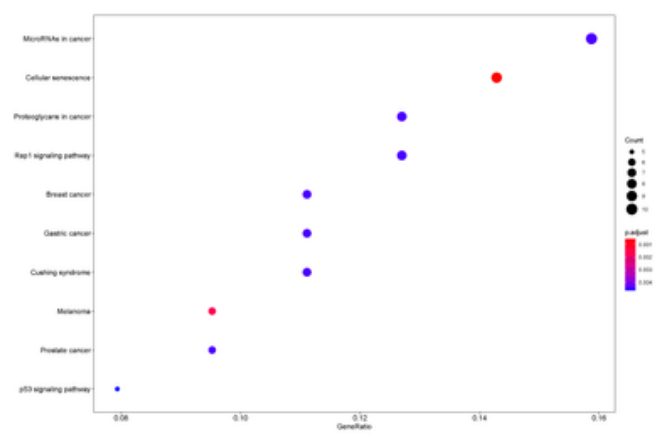

Figure 9 
GESA of functional pathways for DEmRNAs in UCEC. The GO chord (A: CPTAC, E: TCGA) for top 30 DEmRNAs and their GO terms. The GO circle (B: CPTAC, F: TCGA) and the outer circle represented the expression ( $\mid$ log_2FC |) of DEmRNAs in each enriched GO terms. To be specific, red dots on respective GO term indicated the up-regulated DEmRNAs. Blue dots indicated the down-regulated DEmRNAs. The inner circle represented the significance of $G 0$ terms $(\log 10$-adjusted $p$ values). The bar plot (C: CPTAC, G: TCGA) and dot plot (D: CPTAC, H: TCGA) of 10 pathways IncRNA-related DEmRNAs from KEGG pathway analyses. For the bar plot, color intensity represented the significance of ranked functional pathways top to bottom while the horizontal axis represents the enriched mRNAs. For the dot plot, the bubble size was ordered by the number of enriched mRNAs and the color from red to blue also represented its significance.

\section{Supplementary Files}

This is a list of supplementary files associated with this preprint. Click to download.

- supplementtable1.docx

- supplementtable2.docx 